\title{
Protonation of Serine in Gas, Condensed and Microsolvated States in Aqueous Solution
}

\author{
Samantha I. Johnson $*$, Marcel D. Baer ${ }^{\ddagger}$, Simone Raugei ${ }^{\ddagger}$ \\ \$Pacific Northwest National Laboratory, Richland, WA 99354, United States \\ *samanthajo.johnson@pnnl.gov
}

\section{Supporting Information}

\section{Table of Contents}

Table S1 - Gas Phase Basicities Calculated Relative to Water using the BP86 ${ }^{1,2}$ Functional ..................S1

Table S2 - Energies of (MH-M) for different functionals in $\mathrm{kJ} / \mathrm{mol}$. .....................................................S2

Figure S1: Radial Pair Distributions and Coordination Number in Serine in the Condensed Phase ..........S3

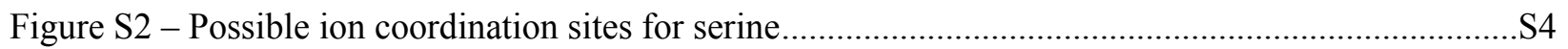

Table S3 $-\mathrm{Na}^{+}$Coordination with Serine with free energy and number of waters ....................................S5

Table S4 $-\mathrm{Cl}^{-}$Coordination with Serine with free energy and number of waters .................................S12

Table S5 - Neutral serine with free energy, number of waters, and tautomeric state .............................S19

Table S6 - Protonated serine with free energy and number of waters ..................................................S39

Figure S3. Average distance between serine center of mass (COM) and ion. .......................................S46

Table S5 - Energy and tautomeric state of the minimum energy structure of neutral serine, protonated serine, deprotonated from amine group serine and deprotonated from carboxylate group serine. ...........S47

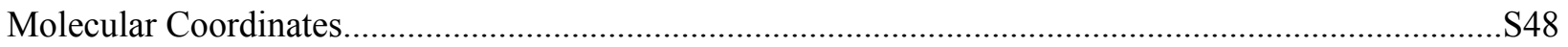


Table S1 - Gas Phase Basicities Calculated Relative to Water using the BP86 ${ }^{1,2}$ Functional

\begin{tabular}{|c|c|c|}
\hline Molecule & Calculated GPB $[\mathrm{kJ} / \mathrm{mol}]$ & $\begin{array}{c}\text { Experimental }{ }^{3} \text { GPB } \\
{[\mathrm{kJ} / \mathrm{mol}]}\end{array}$ \\
\hline $\mathrm{H}_{2} \mathrm{O}$ & 659.8 & 660.0 \\
\hline Pyridine & 900.8 & 898.1 \\
\hline 2-Methylpyridine & 920.5 & 917.3 \\
\hline $2 \mathrm{H}_{2} \mathrm{O}$ & 795.4 & 660.0 \\
\hline dimethylamine & 907.5 & 896.5 \\
\hline Toluene & 733.0 & 756.3 \\
\hline $\mathrm{MeCN}$ & 758.1 & 748.0 \\
\hline $\mathrm{MeOH}$ & 719.2 & 724.5 \\
\hline $\mathrm{CH}_{2} \mathrm{O}$ & 671.5 & 683.3 \\
\hline $\mathrm{NH}_{3}$ & 824.2 & 819.0 \\
\hline $\mathrm{CH}_{2} \mathrm{CH}_{2}$ & 643.5 & 651.5 \\
\hline $\mathrm{CH}_{2} \mathrm{CHCN}$ & 765.3 & 753.7 \\
\hline $\mathrm{CH}_{2} \mathrm{CO}$ & 774.9 & 793.6 \\
\hline $\mathrm{CO}_{2}$ & 507.9 & 515.8 \\
\hline $\mathrm{CS}_{2}$ & 646.4 & 657.7 \\
\hline EtCN & 773.2 & 763.0 \\
\hline $\mathrm{H}_{2} \mathrm{~S}$ & 681.2 & 673.8 \\
\hline HCOOMe & 729.3 & 751.5 \\
\hline NMe3 & 911.7 & 918.1 \\
\hline $\mathrm{Me}_{2} \mathrm{CCH}_{2}$ & 801.2 & 775.6 \\
\hline $\mathrm{Me}_{2} \mathrm{CO}$ & 788.3 & 782.1 \\
\hline $\mathrm{Me}_{2} \mathrm{O}$ & 757.7 & 764.5 \\
\hline $\mathrm{MeCHO}$ & 736.4 & 736.5 \\
\hline $\mathrm{NH}_{2} \mathrm{Et}$ & 883.2 & 878.0 \\
\hline $\mathrm{NH}_{2} \mathrm{Me}$ & 867.3 & 864.5 \\
\hline Propene & 743.5 & 722.7 \\
\hline Average Error & 15.1 & -- \\
\hline
\end{tabular}


Table S2 - Energies of (MH-M) for different functionals in $\mathbf{~ k J / m o l . ~ T h e s e ~ v a l u e s ~ c a n ~ b e ~ u s e d ~ g e n e r a t e ~ a l l ~ G P B ~ v a l u e s . ~}$

\begin{tabular}{|c|c|c|c|c|c|c|c|}
\hline Molecule & BP86-D3 $^{1,2,4,5}$ & B3LYP-D3 ${ }^{1,4-6}$ & $\omega \mathrm{B} 97^{7}$ & M06-D3 $^{8}$ & \begin{tabular}{|c} 
BP86 $^{1,2}$ (large \\
basis opt.)
\end{tabular} & $\omega B 97-D 3^{4,5,7}$ & BLYP-D3 ${ }^{1,4-6}$ \\
\hline $\mathrm{H}_{2} \mathrm{O}$ & -686.5 & -684.9 & -686.5 & -688.2 & -689.1 & -696.6 & -680.9 \\
\hline Pyridine & -927.1 & -961.3 & -925.6 & -918.9 & -931.8 & -944.4 & -927.6 \\
\hline 2-Methylpyridine & -946.9 & -950.3 & -951.8 & -937.7 & -951.7 & -962.5 & -947.1 \\
\hline $2 \mathrm{H}_{2} \mathrm{O}$ & -822.0 & -823.2 & -821.4 & -816.4 & -821.4 & -823.0 & -819.2 \\
\hline dimethylamine & -934.0 & -925.5 & -937.6 & -917.7 & -935.3 & -955.5 & -933.8 \\
\hline Toluene & -759.6 & -759.1 & -758.6 & -754.3 & -758.9 & -751.6 & -764.3 \\
\hline $\mathrm{MeCN}$ & -784.7 & -782.6 & -779.9 & -783.1 & -791.0 & -792.1 & -781.1 \\
\hline $\mathrm{MeOH}$ & -745.8 & -750.7 & -750.0 & -744.4 & -746.1 & -755.8 & -745.4 \\
\hline $\mathrm{CH}_{2} \mathrm{O}$ & -698.0 & -701.1 & -706.8 & -696.1 & -705.3 & -714.6 & -694.3 \\
\hline $\mathrm{NH}_{3}$ & -850.6 & -849.7 & -852.6 & -844.4 & -849.0 & -859.0 & -844.5 \\
\hline $\mathrm{CH}_{2} \mathrm{CH}_{2}$ & -669.8 & -670.6 & -676.0 & -673.0 & -670.7 & -678.1 & -668.8 \\
\hline $\mathrm{CH}_{2} \mathrm{CHCN}$ & -791.6 & -790.8 & -784.1 & -791.0 & -798.0 & -798.7 & -789.6 \\
\hline $\mathrm{CH}_{2} \mathrm{CO}$ & -801.2 & -804.2 & -818.2 & -800.8 & -804.7 & -819.6 & -799.5 \\
\hline $\mathrm{CO}_{2}$ & -534.3 & -530.8 & -532.8 & -528.9 & -537.6 & -540.8 & -531.8 \\
\hline $\mathrm{CS}_{2}$ & -673.0 & -674.4 & -681.2 & -673.8 & -705.8 & -687.4 & -676.0 \\
\hline EtCN & -799.6 & -796.7 & -790.3 & -795.1 & -806.5 & -804.6 & -796.2 \\
\hline $\mathrm{H}_{2} \mathrm{~S}$ & -707.5 & -703.5 & -704.7 & -704.9 & -711.9 & -710.0 & -705.4 \\
\hline HCOOMe & -755.6 & -757.3 & -756.1 & -748.7 & -767.5 & -766.3 & -753.1 \\
\hline NMe3 & -938.0 & -944.0 & -943.2 & -919.8 & -938.7 & -956.4 & -939.5 \\
\hline $\mathrm{Me}_{2} \mathrm{CCH}_{2}$ & -827.8 & -827.3 & -827.7 & -826.4 & -831.1 & -828.3 & -825.3 \\
\hline $\mathrm{Me}_{2} \mathrm{CO}$ & -814.9 & -809.5 & -811.9 & -808.3 & -823.1 & -826.1 & -808.9 \\
\hline $\mathrm{Me}_{2} \mathrm{O}$ & -784.3 & -790.4 & -788.1 & -779.1 & -785.3 & -798.7 & -787.3 \\
\hline $\mathrm{MeCHO}$ & -762.7 & -762.3 & -762.6 & -757.4 & -771.1 & -776.0 & -758.0 \\
\hline $\mathrm{NH}_{2} \mathrm{Et}$ & -909.6 & -910.9 & -909.2 & -898.9 & -910.3 & -918.0 & -907.2 \\
\hline $\mathrm{NH}_{2} \mathrm{Me}$ & -893.7 & -895.5 & -895.7 & -882.5 & -893.8 & -902.5 & -891.0 \\
\hline Propene & -770.1 & -766.0 & -769.7 & -767.9 & -771.4 & -771.9 & -767.8 \\
\hline
\end{tabular}


Figure S1: Radial Pair Distributions and Coordination Number in Serine in the Condensed Phase

Radial pair distribution function $(\mathrm{g}(\mathrm{r})$, solid line) and running coordination number $(\mathrm{n}(\mathrm{r})$, dashed line) between heavy atoms of the serine a) amine nitrogen $(\mathrm{N}), b$ ) hydroxyl oxygen $(\mathrm{OH}), \mathrm{c})$ carboxylic oxygen $(\mathrm{COO})$ and $\mathrm{d}$ ) alpha carbon $(\mathrm{C} \alpha)$ with respect to water oxygen (left panel) and hydrogen (right panel). The neutral form is shown in red and the zwitterionic form in red.
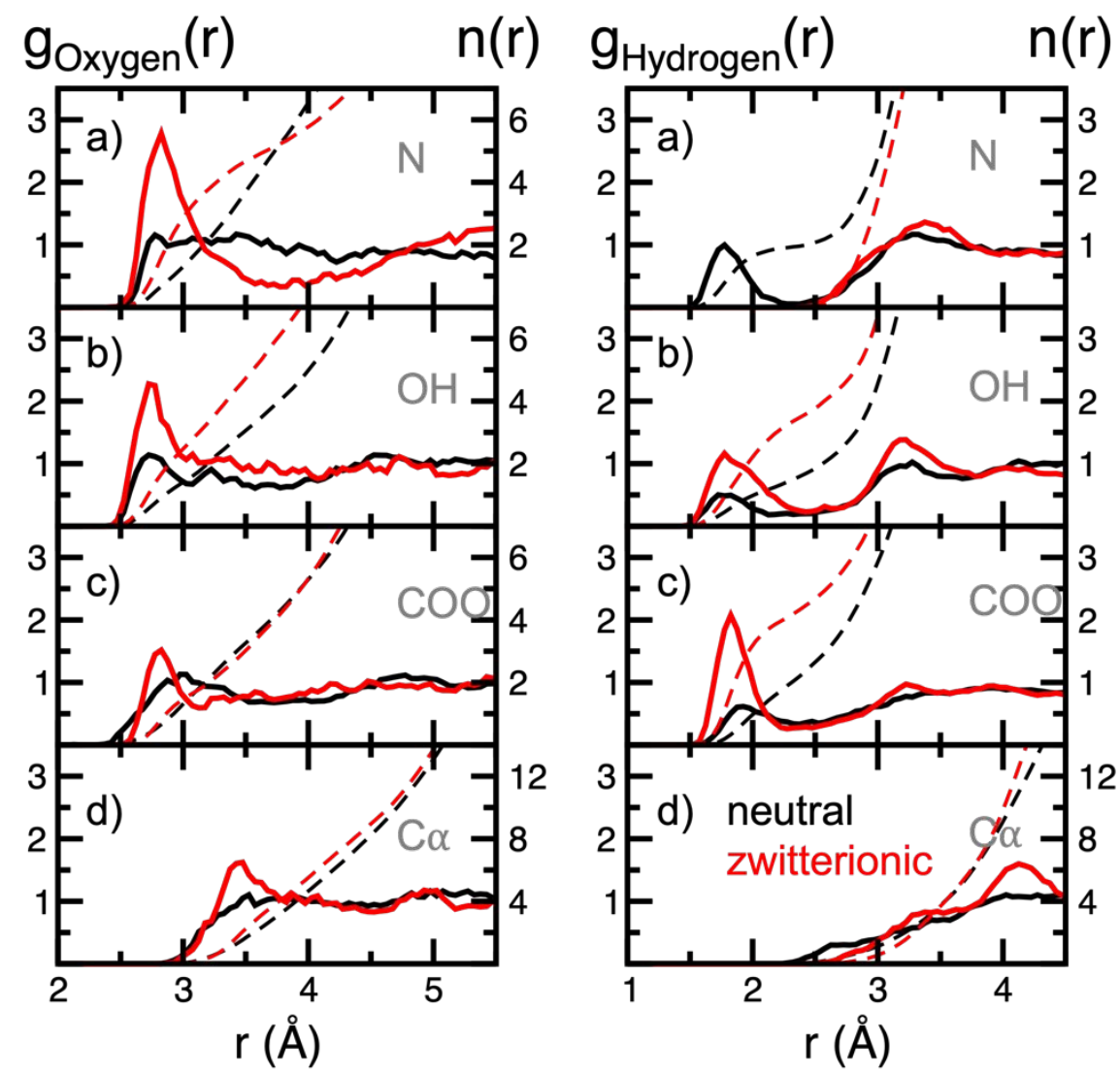


\section{Figure S2 - Possible ion coordination sites for serine}

There are several locations for coordination of $\mathrm{Na}^{+}$, as outlined in Armentrout et al. ${ }^{9,10}$. These sites are unique depending on the tautomer preference. All possible sites are shown here. The preferred site for each geometry is shown below in Tables S3 and S4.

\section{A. $\mathrm{Na}^{+}$coordination}

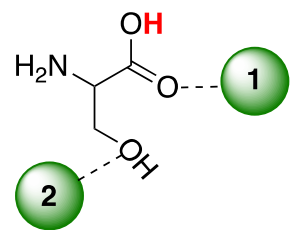

Canonical

B. Cl- coordination

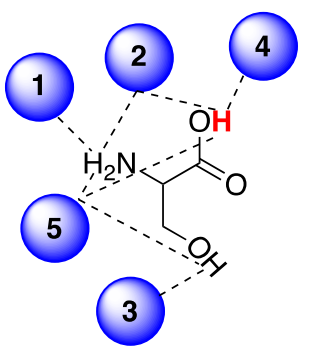

Canonical

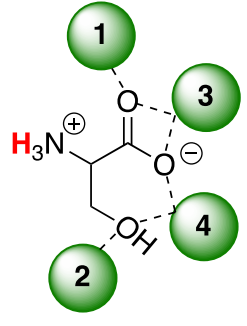

Zwitterion
1. [ $\mathrm{COO}]$

2. $[\mathrm{OH}]$

3. [ $\mathrm{CO} 2-]$

4. $[\mathrm{COO}, \mathrm{OH}]$

5. [ $\mathrm{H} 2 \mathrm{O}]$ - fully solvated by water

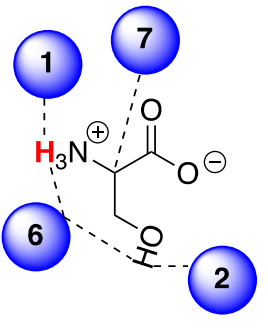

Zwitterion
1. $[\mathrm{NH}]$

2. $[\mathrm{OH}, \mathrm{NH}]$

3. $[\mathrm{OH}]$

4. $[\mathrm{COH}]$

5. [ $\mathrm{COH}, \mathrm{OH}, \mathrm{NH}]$

6. $[\mathrm{OH}, \mathrm{NH}]$

7. $[\mathrm{CH}]$

8. [ $\mathrm{H} 2 \mathrm{O}$ ] - fully solvated by water 
Table $\mathrm{S3}-\mathrm{Na}^{+}$Coordination with Serine with free energy and number of waters

\begin{tabular}{|c|c|c|c|}
\hline Name & Binding & Waters in Cluster & Free Energy [kJ/mol] \\
\hline $\mathrm{Na}$ _serine_watclust_geom6 & {$[\mathrm{COO}, \mathrm{OH}]$} & 1 & -1674217.9 \\
\hline $\mathrm{Na}$ _serine_watclust_geom2 & {$[\mathrm{COO}, \mathrm{OH}]$} & 1 & -1674213 \\
\hline $\mathrm{Na}$ _serine_watclust_geom3 & {$[\mathrm{COO}, \mathrm{OH}]$} & 1 & -1674212.7 \\
\hline $\mathrm{Na}$ _serine_watclust_geom5 & {$[\mathrm{COO}, \mathrm{OH}]$} & 1 & -1674212.7 \\
\hline Na_serine_watclust_geom7 & {$[\mathrm{COO}, \mathrm{OH}]$} & 1 & -1674212.6 \\
\hline $\mathrm{Na}$ _serine_watclust_geom8 & {$[\mathrm{COO}, \mathrm{OH}]$} & 1 & -1674212.3 \\
\hline $\mathrm{Na}$ _serine_watclust_geom4 & {$[\mathrm{COO}, \mathrm{OH}]$} & 1 & -1674211.4 \\
\hline $\mathrm{Na}$ _serine_watclust_geom10 & [CO2- ] & 1 & -1674198 \\
\hline $\mathrm{Na}$ _serine_watclust_geom11 & [CO2- ] & 1 & -1674197.8 \\
\hline $\mathrm{Na}$ _serine_watclust_geom9 & [ $\mathrm{CO} 2-]$ & 1 & -1674196.9 \\
\hline $\mathrm{Na}$ _serine_watclust_geom 12 & [ $\mathrm{COO}]$ & 1 & -1674193.6 \\
\hline $\mathrm{Na}$ _serine_watclust_geom13 & [CO2- ] & 1 & -1674179.7 \\
\hline $\mathrm{Na}$ _serine_watclust_geom17 & {$[\mathrm{COO}, \mathrm{OH}]$} & 2 & -1874976.4 \\
\hline Na_serine_watclust_geom14 & {$[\mathrm{COO}]$} & 2 & -1874976 \\
\hline $\mathrm{Na}$ _serine_watclust_geom18 & {$[\mathrm{COO}, \mathrm{OH}]$} & 2 & -1874975.9 \\
\hline $\mathrm{Na}$ _serine_watclust_geom19 & {$[\mathrm{CO} 2-]$} & 2 & -1874975.4 \\
\hline $\mathrm{Na}$ _serine_watclust_geom15 & {$[\mathrm{COO}, \mathrm{OH}]$} & 2 & -1874969 \\
\hline $\mathrm{Na}$ _serine_watclust_geom16 & {$[\mathrm{COO}, \mathrm{OH}]$} & 2 & -1874968.7 \\
\hline $\mathrm{Na}$ _serine_watclust_geom 21 & {$[\mathrm{COO}]$} & 2 & -1874964.9 \\
\hline $\mathrm{Na}$ _serine_watclust_geom 22 & {$[\mathrm{COO}]$} & 2 & -1874963.1 \\
\hline $\mathrm{Na}$ _serine_watclust_geom 26 & [CO2- ] & 2 & -1874959.1 \\
\hline $\mathrm{Na}$ _serine_watclust_geom 24 & {$[\mathrm{COO}, \mathrm{OH}]$} & 2 & -1874958 \\
\hline $\mathrm{Na}$ _serine_watclust_geom 20 & {$[\mathrm{COO}]$} & 2 & -1874956.5 \\
\hline $\mathrm{Na}$ _serine_watclust_geom 25 & [CO2- ] & 2 & -1874954.9 \\
\hline $\mathrm{Na}$ _serine_watclust_geom 23 & [CO2- ] & 2 & -1874941.8 \\
\hline $\mathrm{Na}$ _serine_watclust_geom 27 & {$[\mathrm{COO}]$} & 3 & -2075734.6 \\
\hline Na_serine_watclust_geom34 & {$[\mathrm{COO}]$} & 3 & -2075726.6 \\
\hline $\mathrm{Na}$ _serine_watclust_geom 30 & {$[\mathrm{COO}, \mathrm{OH}]$} & 3 & -2075723.4 \\
\hline $\mathrm{Na}$ _serine_watclust_geom 33 & {$[\mathrm{COO}, \mathrm{OH}]$} & 3 & -2075721 \\
\hline $\mathrm{Na}$ _serine_watclust_geom 31 & {$[\mathrm{OH}]$} & 3 & -2075720.6 \\
\hline Na_serine_watclust_geom 28 & {$[\mathrm{COO}]$} & 3 & -2075720.1 \\
\hline $\mathrm{Na}$ _serine_watclust_geom 38 & {$[\mathrm{COO}]$} & 3 & -2075719 \\
\hline $\mathrm{Na}$ _serine_watclust_geom 29 & {$[\mathrm{OH}]$} & 3 & -2075718.5 \\
\hline $\mathrm{Na}$ _serine_watclust_geom 37 & [ $\mathrm{COO}]$ & 3 & -2075718.2 \\
\hline $\mathrm{Na}$ _serine_watclust_geom32 & {$[\mathrm{CO} 2, \mathrm{OH}]$} & 3 & -2075717.4 \\
\hline Na_serine_watclust_geom35 & {$[\mathrm{COO}]$} & 3 & -2075716.8 \\
\hline
\end{tabular}




\begin{tabular}{|c|c|c|c|}
\hline $\mathrm{Na}$ _serine_watclust_geom39 & {$[\mathrm{COO}]$} & 3 & -2075712.7 \\
\hline $\mathrm{Na}$ _serine_watclust_geom36 & {$[\mathrm{COO}]$} & 3 & -2075712.1 \\
\hline $\mathrm{Na}$ _serine_watclust_geom41 & {$[\mathrm{OH}]$} & 4 & -2276477.9 \\
\hline $\mathrm{Na}$ _serine_watclust_geom 40 & {$[\mathrm{H} 2 \mathrm{O}]$} & 4 & -2276477.4 \\
\hline $\mathrm{Na}$ _serine_watclust_geom42 & {$[\mathrm{H} 2 \mathrm{O}]$} & 4 & -2276477.1 \\
\hline $\mathrm{Na}$ _serine_watclust_geom 48 & {$[\mathrm{COO}]$} & 4 & -2276477 \\
\hline $\mathrm{Na}$ _serine_watclust_geom45 & {$[\mathrm{COO}]$} & 4 & -2276474.7 \\
\hline $\mathrm{Na}$ _serine_watclust_geom49 & {$[\mathrm{COO}]$} & 4 & -2276474.1 \\
\hline $\mathrm{Na}$ _serine_watclust_geom44 & {$[\mathrm{COO}]$} & 4 & -2276472.6 \\
\hline $\mathrm{Na}$ _serine_watclust_geom50 & {$[\mathrm{COO}]$} & 4 & -2276471.1 \\
\hline $\mathrm{Na}$ _serine_watclust_geom43 & {$[\mathrm{CO} 2-]$} & 4 & -2276470.8 \\
\hline $\mathrm{Na}$ _serine_watclust_geom47 & {$[\mathrm{OH}]$} & 4 & -2276467.3 \\
\hline $\mathrm{Na}$ _serine_watclust_geom46 & {$[\mathrm{COO}]$} & 4 & -2276461.3 \\
\hline $\mathrm{Na}$ _serine_watclust_geom52 & {$[\mathrm{COO}]$} & 4 & -2276461.2 \\
\hline $\mathrm{Na}$ _serine_watclust_geom51 & {$[\mathrm{COO}, \mathrm{OH}]$} & 4 & -2276458.8 \\
\hline $\mathrm{Na}$ _serine_watclust_geom62 & {$[\mathrm{COO}]$} & 5 & -2477233.4 \\
\hline $\mathrm{Na}$ _serine_watclust_geom61 & {$[\mathrm{COO}]$} & 5 & -2477228.6 \\
\hline $\mathrm{Na}$ _serine_watclust_geom54 & {$[\mathrm{CO} 2, \mathrm{OH}]$} & 5 & -2477224.8 \\
\hline $\mathrm{Na}$ _serine_watclust_geom57 & {$[\mathrm{CO} 2-]$} & 5 & -2477222.9 \\
\hline $\mathrm{Na}$ _serine_watclust_geom60 & [CO2- ] & 5 & -2477219.3 \\
\hline $\mathrm{Na}$ _serine_watclust_geom55 & {$[\mathrm{COO}]$} & 5 & -2477219.2 \\
\hline $\mathrm{Na}$ _serine_watclust_geom53 & {$[\mathrm{CO} 2-]$} & 5 & -2477215.1 \\
\hline $\mathrm{Na}$ _serine_watclust_geom58 & {$[\mathrm{OH}]$} & 5 & -2477211.5 \\
\hline Na_serine_watclust_geom63 & {$[\mathrm{COO}]$} & 5 & -2477209.5 \\
\hline $\mathrm{Na}$ _serine_watclust_geom56 & {$[\mathrm{CO} 2-]$} & 5 & -2477208.7 \\
\hline $\mathrm{Na}$ _serine_watclust_geom64 & {$[\mathrm{CO} 2, \mathrm{OH}]$} & 5 & -2477201 \\
\hline $\mathrm{Na}$ _serine_watclust_geom59 & {$[\mathrm{H} 2 \mathrm{O}]$} & 5 & -2477196.6 \\
\hline Na_serine_watclust_geom65 & {$[\mathrm{COO}, \mathrm{OH}]$} & 5 & -2477195 \\
\hline $\mathrm{Na}$ _serine_watclust_geom77 & {$[\mathrm{COO}]$} & 6 & -2677978.8 \\
\hline $\mathrm{Na}$ _serine_watclust_geom78 & {$[\mathrm{OH}]$} & 6 & -2677978.8 \\
\hline $\mathrm{Na}$ _serine_watclust_geom67 & [ $\mathrm{COO}]$ & 6 & -2677978.8 \\
\hline Na_serine_watclust_geom76 & {$[\mathrm{COO}]$} & 6 & -2677976.3 \\
\hline $\mathrm{Na}$ _serine_watclust_geom71 & {$[\mathrm{COO}]$} & 6 & -2677976.2 \\
\hline $\mathrm{Na}$ _serine_watclust_geom69 & {$[\mathrm{CO} 2, \mathrm{OH}]$} & 6 & -2677971.8 \\
\hline $\mathrm{Na}$ _serine_watclust_geom66 & {$[\mathrm{CO} 2-]$} & 6 & -2677970.3 \\
\hline $\mathrm{Na}$ _serine_watclust_geom72 & {$[\mathrm{COO}]$} & 6 & -2677966.5 \\
\hline $\mathrm{Na}$ _serine_watclust_geom75 & [ $\mathrm{H} 2 \mathrm{O}]$ & 6 & -2677965.4 \\
\hline $\mathrm{Na}$ _serine_watclust_geom70 & {$[\mathrm{CO} 2-]$} & 6 & -2677964 \\
\hline $\mathrm{Na}$ _serine_watclust_geom68 & {$[\mathrm{CO} 2-]$} & 6 & -2677961.4 \\
\hline
\end{tabular}




\begin{tabular}{|c|c|c|c|}
\hline $\mathrm{Na}$ _serine_watclust_geom74 & {$[\mathrm{CO} 2-]$} & 6 & -2677952.2 \\
\hline $\mathrm{Na}$ _serine_watclust_geom73 & {$[\mathrm{COO}]$} & 6 & -2677947.9 \\
\hline Na_serine_watclust_geom79 & {$[\mathrm{OH}]$} & 7 & -2878733.7 \\
\hline $\mathrm{Na}$ _serine_watclust_geom81 & {$[\mathrm{COO}]$} & 7 & -2878730.4 \\
\hline $\mathrm{Na}$ serine watclust geom85 & {$[\mathrm{COO}]$} & 7 & -2878729.9 \\
\hline $\mathrm{Na}$ _serine_watclust_geom82 & {$[\mathrm{COO}]$} & 7 & -2878729.5 \\
\hline $\mathrm{Na}$ _serine_watclust_geom87 & {$[\mathrm{COO}]$} & 7 & -2878724.4 \\
\hline $\mathrm{Na}$ _serine_watclust_geom86 & {$[\mathrm{COO}]$} & 7 & -2878724.1 \\
\hline $\mathrm{Na}$ _serine_watclust_geom83 & {$[\mathrm{CO} 2-]$} & 7 & -2878723 \\
\hline $\mathrm{Na}$ _serine_watclust_geom91 & {$[\mathrm{COO}]$} & 7 & -2878720.9 \\
\hline $\mathrm{Na}$ _serine_watclust_geom89 & {$[\mathrm{COO}]$} & 7 & -2878715 \\
\hline $\mathrm{Na}$ _serine_watclust_geom84 & {$[\mathrm{COO}]$} & 7 & -2878711.7 \\
\hline $\mathrm{Na}$ _serine_watclust_geom90 & {$[\mathrm{COO}]$} & 7 & -2878707.6 \\
\hline $\mathrm{Na}$ _serine_watclust_geom 88 & {$[\mathrm{OH}]$} & 7 & -2878705.1 \\
\hline $\mathrm{Na}$ _serine_watclust_geom98 & {$[\mathrm{COO}]$} & 8 & -3079476 \\
\hline Na_serine_watclust_geom 100 & {$[\mathrm{COO}]$} & 8 & -3079475.9 \\
\hline $\mathrm{Na}$ _serine_watclust_geom97 & {$[\mathrm{COO}]$} & 8 & -3079474.1 \\
\hline $\mathrm{Na}$ _serine_watclust_geom92 & {$[\mathrm{COO}]$} & 8 & -3079470.1 \\
\hline Na_serine_watclust_geom 103 & {$[\mathrm{COO}]$} & 8 & -3079466.2 \\
\hline Na_serine_watclust_geom96 & [CO2- ] & 8 & -3079466 \\
\hline Na_serine_watclust_geom101 & {$[\mathrm{COO}]$} & 8 & -3079463.8 \\
\hline Na_serine_watclust_geom102 & {$[\mathrm{COO}]$} & 8 & -3079463.7 \\
\hline Na_serine_watclust_geom99 & {$[\mathrm{COO}]$} & 8 & -3079463.2 \\
\hline $\mathrm{Na}$ _serine_watclust_geom94 & {$[\mathrm{COO}]$} & 8 & -3079461.6 \\
\hline $\mathrm{Na}$ _serine_watclust_geom93 & {$[\mathrm{COO}]$} & 8 & -3079460.3 \\
\hline $\mathrm{Na}$ _serine_watclust_geom95 & {$[\mathrm{COO}]$} & 8 & -3079459.7 \\
\hline Na_serine_watclust_geom104 & [CO2- ] & 8 & -3079459.7 \\
\hline Na_serine_watclust_geom108 & {$[\mathrm{COO}]$} & 9 & -3280225.3 \\
\hline Na_serine_watclust_geom116 & {$[\mathrm{COO}]$} & 9 & -3280223.9 \\
\hline Na_serine_watclust_geom 111 & {$[\mathrm{COO}]$} & 9 & -3280216 \\
\hline Na_serine_watclust_geom117 & [ $\mathrm{COO}]$ & 9 & -3280215.2 \\
\hline Na_serine_watclust_geom109 & [ $\mathrm{H} 2 \mathrm{O}]$ & 9 & -3280214 \\
\hline Na_serine_watclust_geom105 & {$[\mathrm{COO}]$} & 9 & -3280205.9 \\
\hline Na_serine_watclust_geom 113 & {$[\mathrm{COO}]$} & 9 & -3280204.8 \\
\hline Na_serine_watclust_geom106 & {$[\mathrm{COO}]$} & 9 & -3280204.1 \\
\hline Na_serine_watclust_geom114 & {$[\mathrm{COO}]$} & 9 & -3280204 \\
\hline Na_serine_watclust_geom107 & {$[\mathrm{COO}]$} & 9 & -3280203.8 \\
\hline Na_serine_watclust_geom 115 & {$[\mathrm{COO}]$} & 9 & -3280199.1 \\
\hline $\mathrm{Na}$ _serine_watclust_geom 110 & {$[\mathrm{COO}]$} & 9 & -3280196.2 \\
\hline
\end{tabular}




\begin{tabular}{|c|c|c|c|}
\hline Na_serine_watclust_geom 112 & {$[\mathrm{OH}]$} & 9 & -3280196 \\
\hline Na_serine_watclust_geom 126 & {$[\mathrm{COO}]$} & 10 & -3480967.4 \\
\hline Na_serine_watclust_geom 125 & {$[\mathrm{COO}]$} & 10 & -3480964.7 \\
\hline Na_serine_watclust_geom 118 & {$[\mathrm{COO}]$} & 10 & -3480958.5 \\
\hline $\mathrm{Na}$ serine watclust geom 123 & {$[\mathrm{COO}]$} & 10 & -3480956.8 \\
\hline $\mathrm{Na}$ _serine_watclust_geom 120 & {$[\mathrm{CO} 2, \mathrm{OH}]$} & 10 & -3480956.8 \\
\hline $\mathrm{Na}$ _serine_watclust_geom 124 & {$[\mathrm{COO}]$} & 10 & -3480953.8 \\
\hline Na_serine_watclust_geom121 & {$[\mathrm{COO}]$} & 10 & -3480952 \\
\hline Na_serine_watclust_geom119 & {$[\mathrm{COO}]$} & 10 & -3480949.3 \\
\hline Na_serine_watclust_geom 130 & {$[\mathrm{COO}]$} & 10 & -3480946.9 \\
\hline Na_serine_watclust_geom127 & {$[\mathrm{COO}]$} & 10 & -3480944.7 \\
\hline Na_serine_watclust_geom129 & {$[\mathrm{COO}]$} & 10 & -3480940.2 \\
\hline $\mathrm{Na}$ _serine_watclust_geom 122 & [CO2- ] & 10 & -3480935.1 \\
\hline Na_serine_watclust_geom 128 & {$[\mathrm{COO}]$} & 10 & -3480934.4 \\
\hline Na_serine_watclust_geom136 & {$[\mathrm{COO}]$} & 11 & -3681712.9 \\
\hline Na_serine_watclust_geom133 & {$[\mathrm{COO}]$} & 11 & -3681701.4 \\
\hline $\mathrm{Na}$ _serine_watclust_geom 132 & {$[\mathrm{COO}]$} & 11 & -3681697.5 \\
\hline Na_serine_watclust_geom131 & {$[\mathrm{COO}]$} & 11 & -3681696 \\
\hline Na_serine_watclust_geom137 & {$[\mathrm{COO}]$} & 11 & -3681696 \\
\hline Na_serine_watclust_geom 142 & [ $\mathrm{COO}]$ & 11 & -3681694.4 \\
\hline Na_serine_watclust_geom139 & {$[\mathrm{COO}]$} & 11 & -3681694 \\
\hline Na_serine_watclust_geom134 & {$[\mathrm{COO}]$} & 11 & -3681692.5 \\
\hline Na_serine_watclust_geom 138 & {$[\mathrm{COO}]$} & 11 & -3681691.1 \\
\hline Na_serine_watclust_geom141 & {$[\mathrm{COO}]$} & 11 & -3681688 \\
\hline Na_serine_watclust_geom135 & {$[\mathrm{COO}]$} & 11 & -3681683.7 \\
\hline $\mathrm{Na}$ _serine_watclust_geom 140 & {$[\mathrm{CO} 2-]$} & 11 & -3681673.3 \\
\hline Na_serine_watclust_geom145 & {$[\mathrm{COO}]$} & 12 & -3882459.5 \\
\hline Na_serine_watclust_geom153 & {$[\mathrm{COO}]$} & 12 & -3882455.9 \\
\hline Na_serine_watclust_geom146 & {$[\mathrm{COO}]$} & 12 & -3882455.4 \\
\hline Na_serine_watclust_geom147 & [CO2- ] & 12 & -3882454 \\
\hline Na_serine_watclust_geom151 & [ $\mathrm{COO}]$ & 12 & -3882450.2 \\
\hline Na_serine_watclust_geom156 & {$[\mathrm{COO}]$} & 12 & -3882450 \\
\hline Na_serine_watclust_geom155 & {$[\mathrm{COO}]$} & 12 & -3882448.7 \\
\hline $\mathrm{Na}$ _serine_watclust_geom 150 & [ $\mathrm{H} 2 \mathrm{O}]$ & 12 & -3882448.2 \\
\hline Na_serine_watclust_geom149 & {$[\mathrm{COO}]$} & 12 & -3882446.8 \\
\hline Na_serine_watclust_geom144 & {$[\mathrm{COO}]$} & 12 & -3882446.5 \\
\hline Na_serine_watclust_geom 148 & {$[\mathrm{COO}]$} & 12 & -3882446.4 \\
\hline Na_serine_watclust_geom 152 & {$[\mathrm{COO}]$} & 12 & -3882444.9 \\
\hline Na_serine_watclust_geom154 & {$[\mathrm{COO}]$} & 12 & -3882433.3 \\
\hline
\end{tabular}




\begin{tabular}{|c|c|c|c|}
\hline Na_serine_watclust_geom157 & {$[\mathrm{COO}]$} & 13 & -4083210.9 \\
\hline Na_serine_watclust_geom161 & {$[\mathrm{OH}]$} & 13 & -4083203.7 \\
\hline Na_serine_watclust_geom158 & {$[\mathrm{COO}]$} & 13 & -4083202.1 \\
\hline Na_serine_watclust_geom162 & {$[\mathrm{COO}]$} & 13 & -4083199.6 \\
\hline $\mathrm{Na}$ serine watclust geom163 & {$[\mathrm{COO}]$} & 13 & -4083199.1 \\
\hline Na_serine_watclust_geom164 & {$[\mathrm{COO}]$} & 13 & -4083197.1 \\
\hline Na_serine_watclust_geom159 & [ $\mathrm{COO}]$ & 13 & -4083196.6 \\
\hline Na_serine_watclust_geom165 & {$[\mathrm{COO}]$} & 13 & -4083195.6 \\
\hline Na_serine_watclust_geom166 & {$[\mathrm{COO}]$} & 13 & -4083194.3 \\
\hline Na_serine_watclust_geom168 & {$[\mathrm{COO}]$} & 13 & -4083188.1 \\
\hline Na_serine_watclust_geom160 & {$[\mathrm{COO}]$} & 13 & -4083184.3 \\
\hline Na_serine_watclust_geom169 & {$[\mathrm{COO}]$} & 13 & -4083183.4 \\
\hline Na_serine_watclust_geom167 & [ $\mathrm{COO}]$ & 13 & -4083178.7 \\
\hline Na_serine_watclust_geom 179 & {$[\mathrm{COO}]$} & 14 & -4283980.7 \\
\hline Na_serine_watclust_geom 172 & {$[\mathrm{COO}]$} & 14 & -4283959 \\
\hline Na_serine_watclust_geom173 & {$[\mathrm{COO}]$} & 14 & -4283949.8 \\
\hline Na_serine_watclust_geom 180 & [ $\mathrm{COO}]$ & 14 & -4283943.9 \\
\hline Na_serine_watclust_geom178 & [ $\mathrm{COO}]$ & 14 & -4283940.4 \\
\hline Na_serine_watclust_geom174 & [ $\mathrm{COO}]$ & 14 & -4283936.6 \\
\hline Na_serine_watclust_geom 181 & [ H2O ] & 14 & -4283934.1 \\
\hline Na_serine_watclust_geom 175 & {$[\mathrm{COO}]$} & 14 & -4283931.9 \\
\hline Na_serine_watclust_geom176 & {$[\mathrm{COO}]$} & 14 & -4283931.2 \\
\hline Na_serine_watclust_geom 182 & {$[\mathrm{COO}]$} & 14 & -4283928.2 \\
\hline Na_serine_watclust_geom 177 & {$[\mathrm{H} 2 \mathrm{O}]$} & 14 & -4283927.3 \\
\hline Na_serine_watclust_geom 171 & {$[\mathrm{COO}]$} & 14 & -4283924.1 \\
\hline Na_serine_watclust_geom 186 & {$[\mathrm{COO}]$} & 15 & -4484707.6 \\
\hline Na_serine_watclust_geom193 & [ $\mathrm{COO}]$ & 15 & -4484701.5 \\
\hline Na_serine_watclust_geom 188 & {$[\mathrm{COO}]$} & 15 & -4484692.3 \\
\hline Na_serine_watclust_geom 185 & {$[\mathrm{COO}]$} & 15 & -4484690.3 \\
\hline Na_serine_watclust_geom 189 & {$[\mathrm{COO}]$} & 15 & -4484689.4 \\
\hline Na_serine_watclust_geom187 & [ $\mathrm{H} 2 \mathrm{O}]$ & 15 & -4484686.3 \\
\hline Na_serine_watclust_geom191 & {$[\mathrm{H} 2 \mathrm{O}]$} & 15 & -4484684.3 \\
\hline Na_serine_watclust_geom184 & {$[\mathrm{COO}]$} & 15 & -4484682.7 \\
\hline Na_serine_watclust_geom 190 & {$[\mathrm{COO}]$} & 15 & -4484682 \\
\hline Na_serine_watclust_geom192 & {$[\mathrm{COO}]$} & 15 & -4484681.4 \\
\hline Na_serine_watclust_geom183 & {$[\mathrm{COO}]$} & 15 & -4484680.3 \\
\hline Na_serine_watclust_geom195 & [ H2O ] & 15 & -4484680 \\
\hline Na_serine_watclust_geom194 & {$[\mathrm{COO}]$} & 15 & -4484673.2 \\
\hline Na_serine_watclust_geom196 & {$[\mathrm{OH}]$} & 16 & -4685462.1 \\
\hline
\end{tabular}




\begin{tabular}{|c|c|c|c|}
\hline Na_serine_watclust_geom 201 & {$[\mathrm{COO}]$} & 16 & -4685441.8 \\
\hline Na_serine_watclust_geom197 & {$[\mathrm{COO}]$} & 16 & -4685441.1 \\
\hline Na_serine_watclust_geom204 & {$[\mathrm{H} 2 \mathrm{O}]$} & 16 & -4685437.4 \\
\hline Na_serine_watclust_geom198 & {$[\mathrm{COO}]$} & 16 & -4685434.6 \\
\hline $\mathrm{Na}$ serine watclust geom205 & {$[\mathrm{COO}]$} & 16 & -4685431.1 \\
\hline Na_serine_watclust_geom 208 & {$[\mathrm{COO}]$} & 16 & -4685429.2 \\
\hline $\mathrm{Na}$ _serine_watclust_geom 200 & [ $\mathrm{COO}]$ & 16 & -4685425.7 \\
\hline Na_serine_watclust_geom202 & {$[\mathrm{H} 2 \mathrm{O}]$} & 16 & -4685425.3 \\
\hline Na_serine_watclust_geom203 & {$[\mathrm{COO}]$} & 16 & -4685423.8 \\
\hline Na_serine_watclust_geom199 & [ $\mathrm{COO}]$ & 16 & -4685418.9 \\
\hline Na_serine_watclust_geom206 & {$[\mathrm{COO}]$} & 16 & -4685412.3 \\
\hline Na_serine_watclust_geom209 & {$[\mathrm{COO}]$} & 17 & -4886201.3 \\
\hline Na_serine_watclust_geom 212 & [ $\mathrm{COO}]$ & 17 & -4886198.2 \\
\hline Na_serine_watclust_geom 211 & [ H2O ] & 17 & -4886189.2 \\
\hline $\mathrm{Na}$ _serine_watclust_geom 210 & {$[\mathrm{COO}]$} & 17 & -4886182.2 \\
\hline Na_serine_watclust_geom 214 & {$[\mathrm{COO}]$} & 17 & -4886181.4 \\
\hline $\mathrm{Na}$ _serine_watclust_geom 221 & [ $\mathrm{COO}]$ & 17 & -4886181 \\
\hline Na_serine_watclust_geom216 & {$[\mathrm{OH}]$} & 17 & -4886176.9 \\
\hline $\mathrm{Na}$ _serine_watclust_geom 215 & [ $\mathrm{COO}]$ & 17 & -4886173.5 \\
\hline Na_serine_watclust_geom 219 & {$[\mathrm{COO}]$} & 17 & -4886166.6 \\
\hline Na_serine_watclust_geom 218 & {$[\mathrm{COO}]$} & 17 & -4886164 \\
\hline Na_serine_watclust_geom 213 & {$[\mathrm{COO}]$} & 17 & -4886158.4 \\
\hline Na_serine_watclust_geom 220 & {$[\mathrm{COO}]$} & 17 & -4886158.1 \\
\hline Na_serine_watclust_geom 217 & {$[\mathrm{H} 2 \mathrm{O}]$} & 17 & -4886149 \\
\hline Na_serine_watclust_geom 224 & {$[\mathrm{COO}]$} & 18 & -5086934.8 \\
\hline $\mathrm{Na}$ _serine_watclust_geom 222 & [CO2- ] & 18 & -5086932 \\
\hline $\mathrm{Na}$ _serine_watclust_geom 230 & [ $\mathrm{COO}]$ & 18 & -5086921.5 \\
\hline Na_serine_watclust_geom225 & {$[\mathrm{COO}]$} & 18 & -5086919.3 \\
\hline Na_serine_watclust_geom 231 & {$[\mathrm{COO}]$} & 18 & -5086917.8 \\
\hline Na_serine_watclust_geom 228 & {$[\mathrm{COO}]$} & 18 & -5086913.9 \\
\hline Na_serine_watclust_geom 227 & [ $\mathrm{COO}]$ & 18 & -5086911.3 \\
\hline $\mathrm{Na}$ _serine_watclust_geom 226 & {$[\mathrm{OH}]$} & 18 & -5086906.9 \\
\hline Na_serine_watclust_geom 232 & [ H2O ] & 18 & -5086903.9 \\
\hline Na_serine_watclust_geom 234 & [ H2O ] & 18 & -5086900.4 \\
\hline Na_serine_watclust_geom 233 & {$[\mathrm{COO}]$} & 18 & -5086899.8 \\
\hline Na_serine_watclust_geom229 & {$[\mathrm{COO}]$} & 18 & -5086877.5 \\
\hline Na_serine_watclust_geom 238 & [ $\mathrm{COO}]$ & 19 & -5287685.4 \\
\hline Na_serine_watclust_geom 235 & {$[\mathrm{H} 2 \mathrm{O}]$} & 19 & -5287678.3 \\
\hline Na_serine_watclust_geom246 & {$[\mathrm{COO}]$} & 19 & -5287674.8 \\
\hline
\end{tabular}




\begin{tabular}{|c|c|c|c|}
\hline Na_serine_watclust_geom 237 & {$[\mathrm{COO}]$} & 19 & -5287673.6 \\
\hline Na_serine_watclust_geom 236 & {$[\mathrm{H} 2 \mathrm{O}]$} & 19 & -5287670.1 \\
\hline Na_serine_watclust_geom 243 & {$[\mathrm{H} 2 \mathrm{O}]$} & 19 & -5287661.6 \\
\hline Na_serine_watclust_geom 240 & {$[\mathrm{COO}]$} & 19 & -5287653.9 \\
\hline $\mathrm{Na}$ _serine_watclust_geom 242 & {$[\mathrm{H} 2 \mathrm{O}]$} & 19 & -5287652.1 \\
\hline Na_serine_watclust_geom 247 & {$[\mathrm{COO}]$} & 19 & -5287643.3 \\
\hline Na_serine_watclust_geom 245 & {$[\mathrm{COO}]$} & 19 & -5287643 \\
\hline Na_serine_watclust_geom 244 & {$[\mathrm{COO}]$} & 19 & -5287640.7 \\
\hline Na_serine_watclust_geom 249 & [ COO ] & 20 & -5488431.8 \\
\hline Na_serine_watclust_geom 248 & {$[\mathrm{COO}]$} & 20 & -5488408.9 \\
\hline Na_serine_watclust_geom251 & {$[\mathrm{COO}]$} & 20 & -5488407.8 \\
\hline $\mathrm{Na}$ serine_watclust_geom 260 & [ $\mathrm{COO}]$ & 20 & -5488404.1 \\
\hline Na_serine_watclust_geom 250 & {$[\mathrm{COO}]$} & 20 & -5488403.5 \\
\hline Na_serine_watclust_geom 253 & {$[\mathrm{COO}]$} & 20 & -5488400.9 \\
\hline Na_serine_watclust_geom 256 & {$[\mathrm{COO}]$} & 20 & -5488391.3 \\
\hline Na_serine_watclust_geom 257 & {$[\mathrm{OH}]$} & 20 & -5488390.5 \\
\hline Na_serine_watclust_geom 259 & {$[\mathrm{COO}]$} & 20 & -5488389.5 \\
\hline Na_serine_watclust_geom 255 & {$[\mathrm{COO}]$} & 20 & -5488385.8 \\
\hline Na_serine_watclust_geom 258 & {$[\mathrm{COO}]$} & 20 & -5488376.5 \\
\hline
\end{tabular}


Table S4 - $\mathrm{Cl}^{-}$Coordination with Serine with free energy and number of waters

\begin{tabular}{|c|c|c|c|}
\hline Name & Binding & Waters in Cluster & Free Energy [kJ/mol] \\
\hline Cl_serine_sing_geom1 & {$[\mathrm{NH}]$} & 1 & -2457714.3 \\
\hline Cl_serine_sing_geom2 & {$[\mathrm{NH}]$} & 1 & -2457711.6 \\
\hline Cl_serine_sing_geom3 & {$[\mathrm{NH}]$} & 1 & -2457711.0 \\
\hline Cl_serine_sing_geom5 & {$[\mathrm{NH}]$} & 1 & -2457706.7 \\
\hline $\mathrm{Cl}$ _serine_sing_geom8 & {$[\mathrm{NH}]$} & 1 & -2457704.7 \\
\hline Cl_serine_sing_geom6 & {$[\mathrm{NH}]$} & 1 & -2457700.4 \\
\hline Cl_serine_sing_geom9 & {$[\mathrm{NH}]$} & 1 & -2457700.1 \\
\hline Cl_serine_sing_geom 10 & {$[\mathrm{NH}]$} & 1 & -2457696.6 \\
\hline Cl_serine_sing_geom 12 & {$[\mathrm{NH}]$} & 1 & -2457696.3 \\
\hline $\mathrm{Cl}$ _serine_sing_geom 11 & {$[\mathrm{NH}]$} & 1 & -2457694.8 \\
\hline Cl_serine_sing_geom4 & {$[\mathrm{COH}, \mathrm{OH}, \mathrm{NH}]$} & 1 & -2457693.8 \\
\hline Cl_serine_sing_geom 13 & {$[\mathrm{NH}]$} & 1 & -2457693.2 \\
\hline Cl_serine_sing_geom7 & {$[\mathrm{COH}, \mathrm{NH}]$} & 1 & -2457677.6 \\
\hline $\mathrm{Cl}$ _serine_sing_geom 17 & {$[\mathrm{NH}]$} & 2 & -2658581.4 \\
\hline Cl_serine_sing_geom 21 & {$[\mathrm{NH}]$} & 2 & -2658577.6 \\
\hline Cl_serine_sing_geom 14 & {$[\mathrm{NH}]$} & 2 & -2658576.5 \\
\hline Cl_serine_sing_geom 15 & {$[\mathrm{NH}]$} & 2 & -2658574.2 \\
\hline Cl_serine_sing_geom 16 & {$[\mathrm{NH}]$} & 2 & -2658573.2 \\
\hline Cl_serine_sing_geom 22 & {$[\mathrm{NH}]$} & 2 & -2658572.4 \\
\hline Cl_serine_sing_geom 18 & {$[\mathrm{NH}]$} & 2 & -2658567.4 \\
\hline Cl_serine_sing_geom19 & {$[\mathrm{NH}]$} & 2 & -2658564.9 \\
\hline Cl_serine_sing_geom 20 & {$[\mathrm{NH}]$} & 2 & -2658562.3 \\
\hline Cl_serine_sing_geom 23 & {$[\mathrm{NH}]$} & 2 & -2658559.8 \\
\hline Cl_serine_sing_geom 25 & {$[\mathrm{NH}]$} & 2 & -2658559.6 \\
\hline Cl_serine_sing_geom 26 & {$[\mathrm{NH}]$} & 2 & -2658554.2 \\
\hline Cl_serine_sing_geom 24 & {$[\mathrm{COH}, \mathrm{OH}, \mathrm{NH}]$} & 2 & -2658539.8 \\
\hline Cl_serine_sing_geom 27 & {$[\mathrm{OH}, \mathrm{NH}]$} & 3 & -2859456.9 \\
\hline Cl_serine_sing_geom 32 & {$[\mathrm{NH}]$} & 3 & -2859455.0 \\
\hline Cl_serine_sing_geom 28 & {$[\mathrm{NH}]$} & 3 & -2859444.7 \\
\hline Cl_serine_sing_geom 29 & {$[\mathrm{NH}]$} & 3 & -2859440.1 \\
\hline $\mathrm{Cl}$ _serine_sing_geom 30 & {$[\mathrm{NH}]$} & 3 & -2859439.4 \\
\hline Cl_serine_sing_geom 31 & {$[\mathrm{NH}]$} & 3 & -2859439.3 \\
\hline Cl_serine_sing_geom 35 & {$[\mathrm{NH}]$} & 3 & -2859438.6 \\
\hline Cl_serine_sing_geom 33 & {$[\mathrm{NH}]$} & 3 & -2859432.4 \\
\hline Cl_serine_sing_geom 36 & {$[\mathrm{OH}, \mathrm{NH}]$} & 3 & -2859432.3 \\
\hline Cl_serine_sing_geom34 & {$[\mathrm{NH}]$} & 3 & -2859428.9 \\
\hline Cl_serine_sing_geom39 & {$[\mathrm{NH}]$} & 3 & -2859425.2 \\
\hline
\end{tabular}




\begin{tabular}{|c|c|c|c|}
\hline Cl_serine_sing_geom 37 & {$[\mathrm{NH}]$} & 3 & -2859424.6 \\
\hline $\mathrm{Cl}$-serine_sing_geom 38 & {$[\mathrm{NH}]$} & 3 & -2859421.1 \\
\hline Cl_serine_sing_geom 47 & {$[\mathrm{NH}]$} & 4 & -3060326.3 \\
\hline Cl_serine_sing_geom41 & {$[\mathrm{NH}]$} & 4 & -3060322.7 \\
\hline Cl_serine_sing_geom45 & {$[\mathrm{NH}]$} & 4 & -3060321.6 \\
\hline Cl_serine_sing_geom44 & {$[\mathrm{NH}]$} & 4 & -3060320.0 \\
\hline Cl_serine_sing_geom 40 & {$[\mathrm{NH}]$} & 4 & -3060319.3 \\
\hline $\mathrm{Cl}$-serine_sing_geom52 & {$[\mathrm{NH}]$} & 4 & -3060318.0 \\
\hline Cl_serine_sing_geom50 & {$[\mathrm{NH}]$} & 4 & -3060316.8 \\
\hline Cl_serine_sing_geom 42 & {$[\mathrm{NH}]$} & 4 & -3060316.5 \\
\hline Cl_serine_sing_geom43 & {$[\mathrm{NH}]$} & 4 & -3060314.0 \\
\hline Cl_serine_sing_geom 48 & {$[\mathrm{NH}]$} & 4 & -3060305.8 \\
\hline $\mathrm{Cl}$ _serine_sing_geom 46 & {$[\mathrm{NH}]$} & 4 & -3060304.9 \\
\hline Cl_serine_sing_geom51 & {$[\mathrm{NH}]$} & 4 & -3060295.4 \\
\hline Cl_serine_sing_geom49 & {$[\mathrm{NH}]$} & 4 & -3060295.1 \\
\hline Cl_serine_sing_geom63 & {$[\mathrm{NH}]$} & 5 & -3261216.7 \\
\hline Cl_serine_sing_geom65 & {$[\mathrm{NH}]$} & 5 & -3261195.8 \\
\hline Cl_serine_sing_geom56 & {$[\mathrm{NH}]$} & 5 & -3261192.1 \\
\hline Cl_serine_sing_geom58 & {$[\mathrm{NH}]$} & 5 & -3261182.8 \\
\hline Cl_serine_sing_geom54 & [ $\mathrm{H} 2 \mathrm{O}]$ & 5 & -3261181.0 \\
\hline Cl_serine_sing_geom59 & {$[\mathrm{NH}]$} & 5 & -3261180.2 \\
\hline Cl_serine_sing_geom55 & [ H2O ] & 5 & -3261178.8 \\
\hline Cl_serine_sing_geom57 & {$[\mathrm{NH}]$} & 5 & -3261173.2 \\
\hline Cl_serine_sing_geom60 & {$[\mathrm{NH}]$} & 5 & -3261168.2 \\
\hline Cl_serine_sing_geom53 & {$[\mathrm{NH}]$} & 5 & -3261164.8 \\
\hline Cl_serine_sing_geom62 & {$[\mathrm{NH}]$} & 5 & -3261162.7 \\
\hline Cl_serine_sing_geom61 & {$[\mathrm{NH}]$} & 5 & -3261153.6 \\
\hline Cl_serine_sing_geom64 & {$[\mathrm{NH}]$} & 5 & -3261149.2 \\
\hline Cl_serine_sing_geom 74 & [H2O ] & 6 & -3462073.0 \\
\hline Cl_serine_sing_geom67 & {$[\mathrm{NH}]$} & 6 & -3462066.2 \\
\hline Cl_serine_sing_geom66 & {$[\mathrm{NH}]$} & 6 & -3462058.4 \\
\hline Cl_serine_sing_geom69 & {$[\mathrm{NH}]$} & 6 & -3462054.5 \\
\hline Cl_serine_sing_geom78 & {$[\mathrm{NH}]$} & 6 & -3462048.9 \\
\hline $\mathrm{Cl}$ _serine_sing_geom 72 & [ $\mathrm{H} 2 \mathrm{O}]$ & 6 & -3462048.4 \\
\hline Cl_serine_sing_geom75 & {$[\mathrm{NH}]$} & 6 & -3462039.0 \\
\hline Cl_serine_sing_geom68 & {$[\mathrm{NH}]$} & 6 & -3462037.5 \\
\hline Cl_serine_sing_geom76 & [ $\mathrm{H} 2 \mathrm{O}]$ & 6 & -3462036.1 \\
\hline Cl_serine_sing_geom70 & {$[\mathrm{NH}]$} & 6 & -3462035.3 \\
\hline Cl_serine_sing_geom 77 & {$[\mathrm{NH}]$} & 6 & -3462029.2 \\
\hline
\end{tabular}




\begin{tabular}{|c|c|c|c|}
\hline Cl_serine_sing_geom71 & {$[\mathrm{NH}]$} & 6 & -3462024.6 \\
\hline Cl_serine_sing_geom73 & {$[\mathrm{NH}]$} & 6 & -3462013.4 \\
\hline Cl_serine_sing_geom 82 & {$[\mathrm{NH}]$} & 7 & -3662941.9 \\
\hline Cl_serine_sing_geom79 & {$[\mathrm{H} 2 \mathrm{O}]$} & 7 & -3662937.1 \\
\hline Cl_serine_sing_geom83 & {$[\mathrm{NH}]$} & 7 & -3662933.6 \\
\hline Cl_serine_sing_geom80 & {$[\mathrm{NH}]$} & 7 & -3662919.9 \\
\hline Cl_serine_sing_geom85 & [ $\mathrm{H} 2 \mathrm{O}]$ & 7 & -3662917.9 \\
\hline Cl_serine_sing_geom88 & {$[\mathrm{NH}]$} & 7 & -3662911.3 \\
\hline Cl_serine_sing_geom89 & {$[\mathrm{H} 2 \mathrm{O}]$} & 7 & -3662908.5 \\
\hline Cl_serine_sing_geom86 & {$[\mathrm{NH}]$} & 7 & -3662905.1 \\
\hline Cl_serine_sing_geom84 & {$[\mathrm{NH}]$} & 7 & -3662903.5 \\
\hline Cl_serine_sing_geom81 & {$[\mathrm{NH}]$} & 7 & -3662898.5 \\
\hline Cl_serine_sing_geom 90 & {$[\mathrm{NH}]$} & 7 & -3662895.7 \\
\hline Cl_serine_sing_geom91 & {$[\mathrm{NH}]$} & 7 & -3662893.1 \\
\hline Cl_serine_sing_geom87 & {$[\mathrm{NH}]$} & 7 & -3662883.3 \\
\hline Cl_serine_sing_geom93 & {$[\mathrm{NH}]$} & 8 & -3863816.2 \\
\hline Cl_serine_sing_geom92 & {$[\mathrm{NH}]$} & 8 & -3863811.2 \\
\hline Cl_serine_sing_geom95 & {$[\mathrm{NH}]$} & 8 & -3863805.8 \\
\hline Cl_serine_sing_geom98 & {$[\mathrm{OH}]$} & 8 & -3863801.8 \\
\hline Cl_serine_sing_geom97 & {$[\mathrm{NH}]$} & 8 & -3863800.5 \\
\hline Cl_serine_sing_geom101 & {$[\mathrm{NH}]$} & 8 & -3863793.0 \\
\hline Cl_serine_sing_geom96 & {$[\mathrm{H} 2 \mathrm{O}]$} & 8 & -3863790.7 \\
\hline Cl_serine_sing_geom 99 & {$[\mathrm{H} 2 \mathrm{O}]$} & 8 & -3863788.4 \\
\hline Cl_serine_sing_geom 100 & {$[\mathrm{NH}]$} & 8 & -3863779.8 \\
\hline Cl_serine_sing_geom 103 & [H2O ] & 8 & -3863777.7 \\
\hline Cl_serine_sing_geom94 & {$[\mathrm{NH}]$} & 8 & -3863777.4 \\
\hline Cl_serine_sing_geom 104 & {$[\mathrm{H} 2 \mathrm{O}]$} & 8 & -3863749.6 \\
\hline Cl_serine_sing_geom106 & {$[\mathrm{CH}]$} & 9 & -4064680.7 \\
\hline Cl_serine_sing_geom107 & {$[\mathrm{NH}]$} & 9 & -4064678.2 \\
\hline Cl_serine_sing_geom 105 & {$[\mathrm{NH}]$} & 9 & -4064674.4 \\
\hline Cl_serine_sing_geom 111 & {$[\mathrm{CH}]$} & 9 & -4064665.3 \\
\hline Cl_serine_sing_geom 110 & [ $\mathrm{H} 2 \mathrm{O}]$ & 9 & -4064657.1 \\
\hline Cl_serine_sing_geom 108 & {$[\mathrm{NH}]$} & 9 & -4064656.6 \\
\hline Cl_serine_sing_geom 114 & {$[\mathrm{NH}]$} & 9 & -4064656.0 \\
\hline Cl_serine_sing_geom 112 & {$[\mathrm{NH}]$} & 9 & -4064655.3 \\
\hline Cl_serine_sing_geom 117 & {$[\mathrm{NH}]$} & 9 & -4064654.2 \\
\hline Cl_serine_sing_geom 109 & {$[\mathrm{NH}]$} & 9 & -4064653.0 \\
\hline Cl_serine_sing_geom 115 & {$[\mathrm{NH}]$} & 9 & -4064633.6 \\
\hline Cl_serine_sing_geom 116 & {$[\mathrm{NH}]$} & 9 & -4064629.5 \\
\hline
\end{tabular}




\begin{tabular}{|c|c|c|c|}
\hline Cl_serine_sing_geom113 & {$[\mathrm{NH}]$} & 9 & -4064624.6 \\
\hline Cl_serine_sing_geom 123 & {$[\mathrm{H} 2 \mathrm{O}]$} & 10 & -4265556.2 \\
\hline Cl_serine_sing_geom 119 & {$[\mathrm{NH}]$} & 10 & -4265542.9 \\
\hline Cl_serine_sing_geom 118 & {$[\mathrm{OH}]$} & 10 & -4265542.0 \\
\hline Cl_serine_sing_geom 121 & [H2O ] & 10 & -4265534.7 \\
\hline Cl_serine_sing_geom 127 & {$[\mathrm{NH}]$} & 10 & -4265522.7 \\
\hline Cl_serine_sing_geom 120 & {$[\mathrm{NH}]$} & 10 & -4265522.5 \\
\hline Cl_serine_sing_geom124 & {$[\mathrm{OH}]$} & 10 & -4265520.0 \\
\hline Cl_serine_sing_geom 122 & {$[\mathrm{NH}]$} & 10 & -4265512.6 \\
\hline Cl_serine_sing_geom 128 & {$[\mathrm{NH}]$} & 10 & -4265505.8 \\
\hline Cl_serine_sing_geom 125 & {$[\mathrm{H} 2 \mathrm{O}]$} & 10 & -4265503.8 \\
\hline Cl_serine_sing_geom129 & {$[\mathrm{NH}]$} & 10 & -4265499.6 \\
\hline Cl_serine_sing_geom 126 & {$[\mathrm{H} 2 \mathrm{O}]$} & 10 & -4265498.4 \\
\hline Cl_serine_sing_geom 130 & {$[\mathrm{NH}]$} & 10 & -4265478.3 \\
\hline Cl_serine_sing_geom 143 & {$[\mathrm{H} 2 \mathrm{O}]$} & 11 & -4466414.5 \\
\hline Cl_serine_sing_geom133 & {$[\mathrm{NH}]$} & 11 & -4466409.9 \\
\hline Cl_serine_sing_geom134 & {$[\mathrm{NH}]$} & 11 & -4466408.5 \\
\hline Cl_serine_sing_geom 131 & [ H2O ] & 11 & -4466400.2 \\
\hline Cl_serine_sing_geom 132 & {$[\mathrm{H} 2 \mathrm{O}]$} & 11 & -4466393.6 \\
\hline Cl_serine_sing_geom 138 & {$[\mathrm{NH}]$} & 11 & -4466392.3 \\
\hline Cl_serine_sing_geom 135 & {$[\mathrm{NH}]$} & 11 & -4466388.0 \\
\hline Cl_serine_sing_geom139 & {$[\mathrm{H} 2 \mathrm{O}]$} & 11 & -4466387.3 \\
\hline Cl_serine_sing_geom141 & {$[\mathrm{H} 2 \mathrm{O}]$} & 11 & -4466382.9 \\
\hline Cl_serine_sing_geom 137 & {$[\mathrm{NH}]$} & 11 & -4466382.2 \\
\hline Cl_serine_sing_geom140 & {$[\mathrm{NH}]$} & 11 & -4466377.9 \\
\hline Cl_serine_sing_geom142 & {$[\mathrm{NH}]$} & 11 & -4466367.9 \\
\hline Cl_serine_sing_geom 144 & {$[\mathrm{NH}]$} & 12 & -4667278.3 \\
\hline Cl_serine_sing_geom145 & {$[\mathrm{NH}]$} & 12 & -4667268.3 \\
\hline Cl_serine_sing_geom 156 & {$[\mathrm{OH}]$} & 12 & -4667267.6 \\
\hline Cl_serine_sing_geom 147 & {$[\mathrm{NH}]$} & 12 & -4667262.5 \\
\hline Cl_serine_sing_geom 148 & {$[\mathrm{NH}]$} & 12 & -4667260.4 \\
\hline Cl_serine_sing_geom146 & {$[\mathrm{NH}]$} & 12 & -4667256.4 \\
\hline Cl_serine_sing_geom149 & {$[\mathrm{H} 2 \mathrm{O}]$} & 12 & -4667256.2 \\
\hline Cl_serine_sing_geom 150 & [ NH ] & 12 & -4667255.1 \\
\hline Cl_serine_sing_geom152 & {$[\mathrm{H} 2 \mathrm{O}]$} & 12 & -4667252.3 \\
\hline Cl_serine_sing_geom 151 & {$[\mathrm{NH}]$} & 12 & -4667251.6 \\
\hline Cl_serine_sing_geom154 & {$[\mathrm{NH}]$} & 12 & -4667245.4 \\
\hline Cl_serine_sing_geom 153 & {$[\mathrm{NH}]$} & 12 & -4667236.4 \\
\hline Cl_serine_sing_geom155 & {$[\mathrm{NH}]$} & 12 & -4667220.7 \\
\hline
\end{tabular}




\begin{tabular}{|c|c|c|c|}
\hline Cl_serine_sing_geom 157 & {$[\mathrm{NH}]$} & 13 & -4868156.5 \\
\hline Cl_serine_sing_geom159 & {$[\mathrm{OH}]$} & 13 & -4868144.4 \\
\hline Cl_serine_sing_geom 158 & {$[\mathrm{NH}]$} & 13 & -4868143.7 \\
\hline Cl_serine_sing_geom162 & {$[\mathrm{NH}]$} & 13 & -4868131.6 \\
\hline Cl_serine_sing_geom161 & {$[\mathrm{NH}]$} & 13 & -4868126.8 \\
\hline Cl_serine_sing_geom 160 & {$[\mathrm{NH}]$} & 13 & -4868124.8 \\
\hline Cl_serine_sing_geom 164 & {$[\mathrm{NH}]$} & 13 & -4868118.2 \\
\hline Cl_serine_sing_geom 165 & {$[\mathrm{NH}]$} & 13 & -4868114.8 \\
\hline Cl_serine_sing_geom 167 & {$[\mathrm{NH}]$} & 13 & -4868111.5 \\
\hline Cl_serine_sing_geom 166 & [ H2O ] & 13 & -4868107.4 \\
\hline Cl_serine_sing_geom 169 & {$[\mathrm{NH}]$} & 13 & -4868103.9 \\
\hline Cl_serine_sing_geom 168 & {$[\mathrm{NH}]$} & 13 & -4868102.9 \\
\hline Cl_serine_sing_geom 163 & [ $\mathrm{H} 2 \mathrm{O}]$ & 13 & -4868102.3 \\
\hline Cl_serine_sing_geom 170 & {$[\mathrm{NH}]$} & 14 & -5069020.7 \\
\hline Cl_serine_sing_geom 174 & {$[\mathrm{NH}]$} & 14 & -5069005.3 \\
\hline Cl_serine_sing_geom 182 & {$[\mathrm{NH}]$} & 14 & -5068998.6 \\
\hline Cl_serine_sing_geom 175 & {$[\mathrm{NH}]$} & 14 & -5068997.4 \\
\hline Cl_serine_sing_geom 177 & [ H2O ] & 14 & -5068993.4 \\
\hline Cl_serine_sing_geom 172 & {$[\mathrm{NH}]$} & 14 & -5068988.2 \\
\hline Cl_serine_sing_geom 181 & {$[\mathrm{NH}]$} & 14 & -5068987.2 \\
\hline Cl_serine_sing_geom176 & {$[\mathrm{NH}]$} & 14 & -5068979.2 \\
\hline Cl_serine_sing_geom173 & {$[\mathrm{NH}]$} & 14 & -5068973.7 \\
\hline Cl_serine_sing_geom 179 & {$[\mathrm{H} 2 \mathrm{O}]$} & 14 & -5068959.5 \\
\hline Cl_serine_sing_geom 180 & {$[\mathrm{NH}]$} & 14 & -5068957.2 \\
\hline Cl_serine_sing_geom 183 & {$[\mathrm{NH}]$} & 15 & -5269886.6 \\
\hline Cl_serine_sing_geom 184 & {$[\mathrm{NH}]$} & 15 & -5269866.1 \\
\hline Cl_serine_sing_geom 187 & {$[\mathrm{NH}]$} & 15 & -5269864.8 \\
\hline Cl_serine_sing_geom 190 & {$[\mathrm{CH}]$} & 15 & -5269862.2 \\
\hline Cl_serine_sing_geom 188 & [H2O ] & 15 & -5269856.9 \\
\hline Cl_serine_sing_geom 185 & {$[\mathrm{NH}]$} & 15 & -5269856.6 \\
\hline Cl_serine_sing_geom 189 & {$[\mathrm{H} 2 \mathrm{O}]$} & 15 & -5269852.9 \\
\hline Cl_serine_sing_geom191 & {$[\mathrm{NH}]$} & 15 & -5269852.8 \\
\hline Cl_serine_sing_geom 186 & {$[\mathrm{NH}]$} & 15 & -5269846.6 \\
\hline Cl_serine_sing_geom193 & {$[\mathrm{NH}]$} & 15 & -5269839.0 \\
\hline Cl_serine_sing_geom195 & {$[\mathrm{NH}]$} & 15 & -5269833.9 \\
\hline Cl_serine_sing_geom 192 & {$[\mathrm{NH}]$} & 15 & -5269831.9 \\
\hline Cl_serine_sing_geom194 & {$[\mathrm{NH}]$} & 15 & -5269823.0 \\
\hline Cl_serine_sing_geom196 & {$[\mathrm{OH}]$} & 16 & -5470764.1 \\
\hline Cl_serine_sing_geom 202 & {$[\mathrm{NH}]$} & 16 & -5470746.1 \\
\hline
\end{tabular}




\begin{tabular}{|c|c|c|c|}
\hline Cl_serine_sing_geom 201 & [H2O ] & 16 & -5470739.0 \\
\hline Cl_serine_sing_geom198 & {$[\mathrm{NH}]$} & 16 & -5470731.7 \\
\hline Cl_serine_sing_geom 197 & {$[\mathrm{NH}]$} & 16 & -5470729.8 \\
\hline Cl_serine_sing_geom199 & [ H2O ] & 16 & -5470725.7 \\
\hline Cl_serine_sing_geom206 & {$[\mathrm{OH}]$} & 16 & -5470725.3 \\
\hline Cl_serine_sing_geom207 & {$[\mathrm{NH}]$} & 16 & -5470725.0 \\
\hline Cl_serine_sing_geom204 & [ $\mathrm{H} 2 \mathrm{O}]$ & 16 & -5470712.5 \\
\hline Cl_serine_sing_geom208 & {$[\mathrm{NH}]$} & 16 & -5470709.2 \\
\hline Cl_serine_sing_geom 200 & {$[\mathrm{NH}]$} & 16 & -5470699.0 \\
\hline Cl_serine_sing_geom 203 & {$[\mathrm{NH}]$} & 16 & -5470693.5 \\
\hline Cl_serine_sing_geom205 & {$[\mathrm{H} 2 \mathrm{O}]$} & 16 & -5470688.3 \\
\hline Cl_serine_sing_geom 212 & {$[\mathrm{NH}]$} & 17 & -5671621.4 \\
\hline Cl_serine_sing_geom 210 & {$[\mathrm{NH}]$} & 17 & -5671620.8 \\
\hline Cl_serine_sing_geom 213 & {$[\mathrm{NH}]$} & 17 & -5671608.6 \\
\hline Cl_serine_sing_geom 215 & {$[\mathrm{H} 2 \mathrm{O}]$} & 17 & -5671608.0 \\
\hline Cl_serine_sing_geom 221 & {$[\mathrm{H} 2 \mathrm{O}]$} & 17 & -5671601.9 \\
\hline Cl_serine_sing_geom209 & {$[\mathrm{NH}]$} & 17 & -5671600.7 \\
\hline Cl_serine_sing_geom 214 & [ H2O ] & 17 & -5671600.7 \\
\hline Cl_serine_sing_geom 211 & {$[\mathrm{NH}]$} & 17 & -5671597.1 \\
\hline Cl_serine_sing_geom 216 & {$[\mathrm{NH}]$} & 17 & -5671588.9 \\
\hline Cl_serine_sing_geom 218 & [ H2O ] & 17 & -5671577.5 \\
\hline Cl_serine_sing_geom 220 & [ H2O ] & 17 & -5671576.4 \\
\hline Cl_serine_sing_geom 219 & {$[\mathrm{H} 2 \mathrm{O}]$} & 17 & -5671568.6 \\
\hline Cl_serine_sing_geom 217 & {$[\mathrm{NH}]$} & 17 & -5671564.7 \\
\hline Cl_serine_sing_geom 222 & [H2O ] & 18 & -5872481.0 \\
\hline Cl_serine_sing_geom 227 & [H2O ] & 18 & -5872480.8 \\
\hline Cl_serine_sing_geom 223 & {$[\mathrm{NH}]$} & 18 & -5872459.1 \\
\hline Cl_serine_sing_geom 232 & {$[\mathrm{NH}]$} & 18 & -5872447.7 \\
\hline Cl_serine_sing_geom 224 & {$[\mathrm{NH}]$} & 18 & -5872442.6 \\
\hline Cl_serine_sing_geom 229 & {$[\mathrm{NH}]$} & 18 & -5872441.3 \\
\hline Cl_serine_sing_geom 226 & {$[\mathrm{NH}]$} & 18 & -5872440.9 \\
\hline Cl_serine_sing_geom 225 & {$[\mathrm{NH}]$} & 18 & -5872440.2 \\
\hline Cl_serine_sing_geom 231 & {$[\mathrm{NH}]$} & 18 & -5872439.3 \\
\hline Cl_serine_sing_geom 234 & {$[\mathrm{CH}]$} & 18 & -5872438.6 \\
\hline Cl_serine_sing_geom 228 & [H2O ] & 18 & -5872436.4 \\
\hline Cl_serine_sing_geom 233 & {$[\mathrm{CH}]$} & 18 & -5872427.3 \\
\hline Cl_serine_sing_geom 230 & {$[\mathrm{NH}]$} & 18 & -5872417.7 \\
\hline Cl_serine_sing_geom 239 & [H2O ] & 19 & -6073354.3 \\
\hline Cl_serine_sing_geom 245 & {$[\mathrm{H} 2 \mathrm{O}]$} & 19 & -6073343.6 \\
\hline
\end{tabular}




\begin{tabular}{|l|l|l|l|}
\hline Cl_serine_sing_geom244 & {$[\mathrm{H} 2 \mathrm{O}]$} & 19 & -6073331.6 \\
\hline Cl_serine_sing_geom237 & {$[\mathrm{NH}]$} & 19 & -6073328.3 \\
\hline Cl_serine_sing_geom246 & {$[\mathrm{H} 2 \mathrm{O}]$} & 19 & -6073325.3 \\
\hline Cl_serine_sing_geom241 & {$[\mathrm{NH}]$} & 19 & -6073323.8 \\
\hline Cl_serine_sing_geom242 & {$[\mathrm{NH}]$} & 19 & -6073322.4 \\
\hline Cl_serine_sing_geom243 & {$[\mathrm{NH}]$} & 19 & -6073317.4 \\
\hline Cl_serine_sing_geom247 & {$[\mathrm{OH}]$} & 19 & -6073314.9 \\
\hline Cl_serine_sing_geom238 & {$[\mathrm{NH}]$} & 19 & -6073309.7 \\
\hline Cl_serine_sing_geom240 & {$[\mathrm{NH}]$} & 19 & -6073293.1 \\
\hline C1_serine_sing_geom248 & {$[\mathrm{H} 2 \mathrm{O}]$} & 20 & -6274221.9 \\
\hline Cl_serine_sing_geom250 & {$[\mathrm{H} 2 \mathrm{O}]$} & 20 & -6274221.1 \\
\hline Cl_serine_sing_geom257 & {$[\mathrm{OH}]$} & 20 & -6274203.6 \\
\hline Cl_serine_sing_geom251 & {$[\mathrm{NH}]$} & 20 & -6274201.4 \\
\hline Cl_serine_sing_geom249 & {$[\mathrm{H} 2 \mathrm{O}]$} & 20 & -6274192.5 \\
\hline Cl_serine_sing_geom256 & {$[\mathrm{NH}]$} & 20 & -6274187.0 \\
\hline Cl_serine_sing_geom255 & {$[\mathrm{NH}]$} & 20 & -6274186.4 \\
\hline Cl_serine_sing_geom258 & {$[\mathrm{NH}]$} & 20 & -6274184.8 \\
\hline Cl_serine_sing_geom260 & {$[\mathrm{H} 2 \mathrm{O}]$} & -6274177.8 \\
\hline Cl_serine_sing_geom259 & {$[\mathrm{NH}]$} & 20 & -6274175.1 \\
\hline Cl_serine_sing_geom252 & {$[\mathrm{H} 2 \mathrm{O}]$} & 20 & -6274174.8 \\
\hline Cl_serine_sing_geom254 & {$[\mathrm{NH}]$} & 20 & -6274167.4 \\
\hline
\end{tabular}


Table S5 - Neutral serine with free energy, number of waters, and tautomeric state

\begin{tabular}{|c|c|c|c|}
\hline File Name & Free Energy [kJ/mol] & Waters (n) & Tautomeric State \\
\hline serine_xwat_sing_geom1 & -1248431.269 & 1 & 0 \\
\hline serine_xwat_sing_geom2 & -1248428.799 & 1 & 0 \\
\hline serine1_neutral_geom1 & -1248428.773 & 1 & 0 \\
\hline serine1_neutral_geom9 & -1248428.667 & 1 & 0 \\
\hline serine_xwat_sing_geom3 & -1248428.661 & 1 & 0 \\
\hline serine_xwat_sing_geom4 & -1248426.620 & 1 & 0 \\
\hline serine2_neutral_geom1 & -1248425.346 & 1 & 0 \\
\hline serine1_neutral_geom2 & -1248425.207 & 1 & 0 \\
\hline serine_xwat_sing_geom9 & -1248425.156 & 1 & 0 \\
\hline serine_xwat_sing_geom8 & -1248425.096 & 1 & 0 \\
\hline serine_xwat_sing_geom6 & -1248425.091 & 1 & 0 \\
\hline serine1_neutral_geom4 & -1248425.089 & 1 & 0 \\
\hline serine1_neutral_geom7 & -1248425.086 & 1 & 0 \\
\hline serine1_neutral_geom3 & -1248425.058 & 1 & 0 \\
\hline serine_xwat_sing_geom5 & -1248425.037 & 1 & 0 \\
\hline serine2_neutral_geom2 & -1248424.516 & 1 & 0 \\
\hline serine1_neutral_geom5 & -1248424.422 & 1 & 0 \\
\hline serine_xwat_sing_geom7 & -1248424.096 & 1 & 0 \\
\hline serine2_neutral_geom3 & -1248423.580 & 1 & 0 \\
\hline serine_xwat_sing_geom 13 & -1248423.122 & 1 & 0 \\
\hline serine2_neutral_geom11 & -1248422.959 & 1 & 0 \\
\hline serine2_neutral_geom5 & -1248421.385 & 1 & 0 \\
\hline serine2_neutral_geom10 & -1248421.118 & 1 & 0 \\
\hline serine1_neutral_geom6 & -1248419.296 & 1 & 0 \\
\hline serine2_neutral_geom6 & -1248418.071 & 1 & 0 \\
\hline serine2_neutral_geom7 & -1248418.043 & 1 & 0 \\
\hline serine2_neutral_geom4 & -1248417.990 & 1 & 0 \\
\hline serine2_neutral_geom9 & -1248415.559 & 1 & 0 \\
\hline serine2_neutral_geom12 & -1248415.494 & 1 & 0 \\
\hline serine2_neutral_geom8 & -1248415.463 & 1 & 0 \\
\hline serine_xwat_sing_geom 10 & -1248414.593 & 1 & 0 \\
\hline serine_xwat_sing_geom 12 & -1248414.393 & 1 & 0 \\
\hline serine1_neutral_geom8 & -1248410.149 & 1 & 0 \\
\hline serine2_neutral_geom13 & -1248407.811 & 1 & 0 \\
\hline serine2_neutral_geom14 & -1449183.245 & 2 & 0 \\
\hline serine1_neutral_geom10 & -1449174.820 & 2 & 0 \\
\hline serine_xwat_sing_geom14 & -1449174.579 & 2 & 0 \\
\hline
\end{tabular}




\begin{tabular}{|c|c|c|c|}
\hline serine_xwat_sing_geom 16 & -1449173.325 & 2 & 0 \\
\hline serine1_neutral_geom13 & -1449173.098 & 2 & 0 \\
\hline serine_xwat_sing_geom19 & -1449172.490 & 2 & 0 \\
\hline serine_xwat_sing_geom 15 & -1449168.220 & 2 & 0 \\
\hline serine1_neutral_geom11 & -1449168.193 & 2 & 0 \\
\hline serine2_neutral_geom 23 & -1449166.121 & 2 & 0 \\
\hline serine_xwat_sing_geom 18 & -1449166.108 & 2 & 0 \\
\hline serine2_neutral_geom 25 & -1449166.076 & 2 & 0 \\
\hline serine2_neutral_geom15 & -1449165.617 & 2 & 0 \\
\hline serine1_neutral_geom15 & -1449163.213 & 2 & 0 \\
\hline serine2_neutral_geom16 & -1449163.071 & 2 & 0 \\
\hline serine_xwat_sing_geom 20 & -1449162.947 & 2 & 0 \\
\hline serine2_neutral_geom18 & -1449162.791 & 2 & 0 \\
\hline serine2_neutral_geom 20 & -1449161.808 & 2 & 0 \\
\hline serine_xwat_sing_geom 21 & -1449161.758 & 2 & 0 \\
\hline serine2_neutral_geom17 & -1449161.182 & 2 & 0 \\
\hline serine1_neutral_geom22 & -1449160.136 & 2 & 0 \\
\hline serine2_neutral_geom19 & -1449158.681 & 2 & 0 \\
\hline serine2_neutral_geom 26 & -1449158.649 & 2 & 0 \\
\hline serine_xwat_sing_geom 17 & -1449158.620 & 2 & 0 \\
\hline serine1_neutral_geom16 & -1449158.544 & 2 & 0 \\
\hline serine1_neutral_geom12 & -1449158.309 & 2 & 0 \\
\hline serine1_neutral_geom17 & -1449157.707 & 2 & 0 \\
\hline serine1_neutral_geom14 & -1449156.993 & 2 & 0 \\
\hline serine2_neutral_geom22 & -1449155.228 & 2 & 0 \\
\hline serine1_neutral_geom 20 & -1449155.035 & 2 & 0 \\
\hline serine_xwat_sing_geom 22 & -1449154.815 & 2 & 0 \\
\hline serine_xwat_sing_geom 24 & -1449152.423 & 2 & 0 \\
\hline serine_xwat_sing_geom 23 & -1449152.362 & 2 & 0 \\
\hline serine1_neutral_geom18 & -1449152.325 & 2 & 0 \\
\hline serine1_neutral_geom19 & -1449152.282 & 2 & 0 \\
\hline serine1_neutral_geom 21 & -1449152.210 & 2 & 0 \\
\hline serine_xwat_sing_geom 26 & -1449152.129 & 2 & 0 \\
\hline serine2_neutral_geom24 & -1449151.915 & 2 & 0 \\
\hline serine2_neutral_geom21 & -1449150.535 & 2 & 0 \\
\hline serine_xwat_sing_geom 25 & -1449146.563 & 2 & 0 \\
\hline serine1_neutral_geom25 & -1649918.937 & 3 & 0 \\
\hline serine2_neutral_geom 28 & -1649917.499 & 3 & 0 \\
\hline serine2_neutral_geom27 & -1649915.211 & 3 & 0 \\
\hline
\end{tabular}




\begin{tabular}{|c|c|c|c|}
\hline serine2_neutral_geom29 & -1649912.879 & 3 & 0 \\
\hline serine1_neutral_geom27 & -1649911.694 & 3 & 0 \\
\hline serine1_neutral_geom23 & -1649911.587 & 3 & 0 \\
\hline serine_xwat_sing_geom 28 & -1649911.346 & 3 & 0 \\
\hline serine1_neutral_geom24 & -1649911.267 & 3 & 0 \\
\hline serine2_neutral_geom31 & -1649910.099 & 3 & 0 \\
\hline serine_xwat_sing_geom 27 & -1649909.733 & 3 & 0 \\
\hline serine2_neutral_geom30 & -1649909.705 & 3 & 0 \\
\hline serine_xwat_sing_geom 29 & -1649908.331 & 3 & 0 \\
\hline serine_xwat_sing_geom 32 & -1649906.701 & 3 & 0 \\
\hline serine2_neutral_geom32 & -1649906.529 & 3 & 0 \\
\hline serine1_neutral_geom30 & -1649905.691 & 3 & 0 \\
\hline serine1_neutral_geom 26 & -1649905.036 & 3 & 0 \\
\hline serine1_neutral_geom29 & -1649903.360 & 3 & 0 \\
\hline serine2_neutral_geom34 & -1649903.241 & 3 & 0 \\
\hline serine_xwat_sing_geom 31 & -1649902.139 & 3 & 0 \\
\hline serine_xwat_sing_geom 30 & -1649901.815 & 3 & 0 \\
\hline serine2_neutral_geom36 & -1649899.790 & 3 & 0 \\
\hline serine2_neutral_geom33 & -1649899.760 & 3 & 0 \\
\hline serine_xwat_sing_geom 33 & -1649899.758 & 3 & 0 \\
\hline serine2_neutral_geom35 & -1649899.502 & 3 & 0 \\
\hline serine2_neutral_geom 38 & -1649898.118 & 3 & 0 \\
\hline serine1_neutral_geom35 & -1649897.978 & 3 & 0 \\
\hline serine1_neutral_geom32 & -1649897.633 & 3 & 0 \\
\hline serine_xwat_sing_geom 37 & -1649897.146 & 3 & 0 \\
\hline serine1_neutral_geom34 & -1649897.109 & 3 & 0 \\
\hline serine_xwat_sing_geom 38 & -1649896.805 & 3 & 0 \\
\hline serine2_neutral_geom37 & -1649895.177 & 3 & 0 \\
\hline serine2_neutral_geom39 & -1649895.055 & 3 & 0 \\
\hline serine_xwat_sing_geom39 & -1649894.878 & 3 & 0 \\
\hline serine_xwat_sing_geom 34 & -1649894.776 & 3 & 0 \\
\hline serine1_neutral_geom31 & -1649894.734 & 3 & 0 \\
\hline serine1_neutral_geom33 & -1649891.236 & 3 & 0 \\
\hline serine_xwat_sing_geom 36 & -1649890.680 & 3 & 0 \\
\hline serine1_neutral_geom 28 & -1649890.141 & 3 & 0 \\
\hline serine_xwat_sing_geom 35 & -1649888.373 & 3 & 0 \\
\hline serine2_neutral_geom40 & -1850661.648 & 4 & 0 \\
\hline serine2_neutral_geom41 & -1850656.843 & 4 & 0 \\
\hline serine2_neutral_geom46 & -1850650.278 & 4 & 0 \\
\hline
\end{tabular}




\begin{tabular}{|c|c|c|c|}
\hline serine2_neutral_geom42 & -1850649.885 & 4 & 0 \\
\hline serine2_neutral_geom44 & -1850647.739 & 4 & 0 \\
\hline serine2_neutral_geom50 & -1850646.892 & 4 & 0 \\
\hline serine_xwat_sing_geom 40 & -1850646.778 & 4 & 0 \\
\hline serine2_neutral_geom51 & -1850646.777 & 4 & 0 \\
\hline serine2_neutral_geom43 & -1850646.764 & 4 & 0 \\
\hline serine_xwat_sing_geom41 & -1850646.002 & 4 & 0 \\
\hline serine1_neutral_geom36 & -1850645.256 & 4 & 0 \\
\hline serine_xwat_sing_geom 45 & -1850644.120 & 4 & 0 \\
\hline serine1_neutral_geom37 & -1850643.038 & 4 & 0 \\
\hline serine1_neutral_geom38 & -1850640.128 & 4 & 0 \\
\hline serine_xwat_sing_geom 48 & -1850639.050 & 4 & 0 \\
\hline serine1_neutral_geom40 & -1850638.925 & 4 & 0 \\
\hline serine_xwat_sing_geom 42 & -1850638.396 & 4 & 0 \\
\hline serine_xwat_sing_geom49 & -1850638.206 & 4 & 0 \\
\hline serine_xwat_sing_geom47 & -1850638.065 & 4 & 0 \\
\hline serine1_neutral_geom44 & -1850638.044 & 4 & 0 \\
\hline serine1_neutral_geom39 & -1850637.946 & 4 & 0 \\
\hline serine2_neutral_geom48 & -1850637.730 & 4 & 0 \\
\hline serine2_neutral_geom45 & -1850637.624 & 4 & 0 \\
\hline serine1_neutral_geom48 & -1850637.456 & 4 & 0 \\
\hline serine1_neutral_geom43 & -1850636.796 & 4 & 0 \\
\hline serine_xwat_sing_geom52 & -1850636.739 & 4 & 0 \\
\hline serine_xwat_sing_geom50 & -1850636.671 & 4 & 0 \\
\hline serine_xwat_sing_geom43 & -1850635.366 & 4 & 0 \\
\hline serine1_neutral_geom41 & -1850635.165 & 4 & 0 \\
\hline serine_xwat_sing_geom 44 & -1850635.116 & 4 & 0 \\
\hline serine1_neutral_geom45 & -1850634.713 & 4 & 0 \\
\hline serine_xwat_sing_geom46 & -1850634.142 & 4 & 0 \\
\hline serine2_neutral_geom49 & -1850633.185 & 4 & 0 \\
\hline serine1_neutral_geom46 & -1850631.158 & 4 & 0 \\
\hline serine1_neutral_geom47 & -1850629.653 & 4 & 0 \\
\hline serine2_neutral_geom47 & -1850625.127 & 4 & 0 \\
\hline serine1_neutral_geom42 & -1850624.960 & 4 & 0 \\
\hline serine_xwat_sing_geom51 & -1850624.907 & 4 & 0 \\
\hline serine2_neutral_geom53 & -2051405.829 & 5 & 2 \\
\hline serine2_neutral_geom64 & -2051405.520 & 5 & 2 \\
\hline serine2_neutral_geom54 & -2051396.071 & 5 & 2 \\
\hline serine1_neutral_geom50 & -2051393.869 & 5 & 2 \\
\hline
\end{tabular}




\begin{tabular}{|c|c|c|c|}
\hline serine2_neutral_geom57 & -2051390.699 & 5 & 0 \\
\hline serine2_neutral_geom63 & -2051387.581 & 5 & 0 \\
\hline serine1_neutral_geom49 & -2051386.785 & 5 & 2 \\
\hline serine2_neutral_geom59 & -2051384.865 & 5 & 0 \\
\hline serine2_neutral_geom58 & -2051382.007 & 5 & 2 \\
\hline serine1_neutral_geom54 & -2051381.846 & 5 & 0 \\
\hline serine_xwat_sing_geom57 & -2051381.779 & 5 & 0 \\
\hline serine2_neutral_geom55 & -2051381.595 & 5 & 0 \\
\hline serine_xwat_sing_geom62 & -2051381.162 & 5 & 0 \\
\hline serine1_neutral_geom56 & -2051380.484 & 5 & 0 \\
\hline serine_xwat_sing_geom61 & -2051379.962 & 5 & 0 \\
\hline serine1_neutral_geom53 & -2051379.880 & 5 & 0 \\
\hline serine_xwat_sing_geom63 & -2051379.607 & 5 & 0 \\
\hline serine1_neutral_geom51 & -2051379.466 & 5 & 0 \\
\hline serine1_neutral_geom58 & -2051379.308 & 5 & 0 \\
\hline serine_xwat_sing_geom54 & -2051378.864 & 5 & 0 \\
\hline serine_xwat_sing_geom65 & -2051378.477 & 5 & 0 \\
\hline serine_xwat_sing_geom59 & -2051378.418 & 5 & 0 \\
\hline serine_xwat_sing_geom55 & -2051378.177 & 5 & 0 \\
\hline serine1_neutral_geom55 & -2051378.110 & 5 & 0 \\
\hline serine2_neutral_geom61 & -2051377.463 & 5 & 0 \\
\hline serine_xwat_sing_geom58 & -2051377.029 & 5 & 0 \\
\hline serine1_neutral_geom57 & -2051376.155 & 5 & 0 \\
\hline serine1_neutral_geom61 & -2051375.137 & 5 & 0 \\
\hline serine2_neutral_geom60 & -2051374.053 & 5 & 0 \\
\hline serine2_neutral_geom62 & -2051373.955 & 5 & 0 \\
\hline serine_xwat_sing_geom53 & -2051373.915 & 5 & 0 \\
\hline serine_xwat_sing_geom56 & -2051373.679 & 5 & 0 \\
\hline serine1_neutral_geom52 & -2051372.510 & 5 & 0 \\
\hline serine_xwat_sing_geom64 & -2051372.223 & 5 & 0 \\
\hline serine1_neutral_geom59 & -2051371.605 & 5 & 0 \\
\hline serine1_neutral_geom60 & -2051367.710 & 5 & 0 \\
\hline serine_xwat_sing_geom60 & -2051366.092 & 5 & 0 \\
\hline serine2_neutral_geom65 & -2051364.140 & 5 & 0 \\
\hline serine1_neutral_geom69 & -2252150.767 & 6 & 2 \\
\hline serine1_neutral_geom67 & -2252149.784 & 6 & 2 \\
\hline serine_xwat_sing_geom77 & -2252148.289 & 6 & 2 \\
\hline serine2_neutral_geom66 & -2252146.249 & 6 & 0 \\
\hline serine1_neutral_geom62 & -2252145.856 & 6 & 2 \\
\hline
\end{tabular}




\begin{tabular}{|c|c|c|c|}
\hline serine2_neutral_geom68 & -2252143.557 & 6 & 2 \\
\hline serine2_neutral_geom77 & -2252140.146 & 6 & 2 \\
\hline serine1_neutral_geom70 & -2252139.859 & 6 & 2 \\
\hline serine2_neutral_geom67 & -2252138.418 & 6 & 2 \\
\hline serine2_neutral_geom71 & -2252138.017 & 6 & 2 \\
\hline serine1_neutral_geom64 & -2252134.421 & 6 & 2 \\
\hline serine2_neutral_geom72 & -2252133.058 & 6 & 2 \\
\hline serine_xwat_sing_geom66 & -2252132.087 & 6 & 2 \\
\hline serine1_neutral_geom73 & -2252131.591 & 6 & 2 \\
\hline serine2_neutral_geom78 & -2252131.582 & 6 & 2 \\
\hline serine2_neutral_geom69 & -2252131.001 & 6 & 2 \\
\hline serine_xwat_sing_geom67 & -2252130.297 & 6 & 2 \\
\hline serine2_neutral_geom76 & -2252125.421 & 6 & 2 \\
\hline serine1_neutral_geom63 & -2252124.842 & 6 & 2 \\
\hline serine2_neutral_geom75 & -2252119.109 & 6 & 0 \\
\hline serine2_neutral_geom73 & -2252118.635 & 6 & 0 \\
\hline serine1_neutral_geom68 & -2252118.489 & 6 & 0 \\
\hline serine_xwat_sing_geom68 & -2252118.226 & 6 & 0 \\
\hline serine2_neutral_geom70 & -2252117.251 & 6 & 1 \\
\hline serine2_neutral_geom74 & -2252116.177 & 6 & 2 \\
\hline serine_xwat_sing_geom73 & -2252113.848 & 6 & 0 \\
\hline serine_xwat_sing_geom71 & -2252113.773 & 6 & 0 \\
\hline serine_xwat_sing_geom78 & -2252113.342 & 6 & 0 \\
\hline serine_xwat_sing_geom74 & -2252111.126 & 6 & 0 \\
\hline serine_xwat_sing_geom75 & -2252111.020 & 6 & 0 \\
\hline serine_xwat_sing_geom76 & -2252108.113 & 6 & 0 \\
\hline serine_xwat_sing_geom72 & -2252107.936 & 6 & 0 \\
\hline serine_xwat_sing_geom70 & -2252106.194 & 6 & 0 \\
\hline serine1_neutral_geom71 & -2252105.385 & 6 & 0 \\
\hline serine_xwat_sing_geom69 & -2252103.117 & 6 & 0 \\
\hline serine1_neutral_geom74 & -2252101.218 & 6 & 0 \\
\hline serine1_neutral_geom72 & -2252100.423 & 6 & 0 \\
\hline serine1_neutral_geom66 & -2252100.285 & 6 & 0 \\
\hline serine2_neutral_geom80 & -2452898.084 & 7 & 2 \\
\hline serine_xwat_sing_geom87 & -2452894.845 & 7 & 2 \\
\hline serine1_neutral_geom75 & -2452893.223 & 7 & 2 \\
\hline serine2_neutral_geom79 & -2452891.791 & 7 & 2 \\
\hline serine1_neutral_geom76 & -2452891.707 & 7 & 2 \\
\hline serine1_neutral_geom86 & -2452889.898 & 7 & 2 \\
\hline
\end{tabular}




\begin{tabular}{|c|c|c|c|}
\hline serine2_neutral_geom83 & -2452884.888 & 7 & 2 \\
\hline serine_xwat_sing_geom 83 & -2452884.282 & 7 & 2 \\
\hline serine2_neutral_geom82 & -2452883.974 & 7 & 2 \\
\hline serine1_neutral_geom82 & -2452883.498 & 7 & 2 \\
\hline serine2_neutral_geom81 & -2452880.296 & 7 & 2 \\
\hline serine2_neutral_geom87 & -2452879.180 & 7 & 2 \\
\hline serine_xwat_sing_geom79 & -2452877.624 & 7 & 2 \\
\hline serine2_neutral_geom89 & -2452875.496 & 7 & 2 \\
\hline serine2_neutral_geom84 & -2452875.022 & 7 & 2 \\
\hline serine2_neutral_geom85 & -2452873.959 & 7 & 2 \\
\hline serine2_neutral_geom90 & -2452873.147 & 7 & 2 \\
\hline serine1_neutral_geom78 & -2452872.765 & 7 & 2 \\
\hline serine_xwat_sing_geom85 & -2452872.407 & 7 & 0 \\
\hline serine_xwat_sing_geom 80 & -2452871.812 & 7 & 2 \\
\hline serine1_neutral_geom77 & -2452871.525 & 7 & 2 \\
\hline serine_xwat_sing_geom81 & -2452870.565 & 7 & 2 \\
\hline serine_xwat_sing_geom91 & -2452870.082 & 7 & 2 \\
\hline serine1_neutral_geom84 & -2452869.134 & 7 & 2 \\
\hline serine_xwat_sing_geom 86 & -2452868.869 & 7 & 2 \\
\hline serine2_neutral_geom86 & -2452866.041 & 7 & 2 \\
\hline serine1_neutral_geom80 & -2452865.651 & 7 & 0 \\
\hline serine2_neutral_geom91 & -2452862.491 & 7 & 0 \\
\hline serine2_neutral_geom88 & -2452860.293 & 7 & 2 \\
\hline serine1_neutral_geom81 & -2452858.762 & 7 & 0 \\
\hline serine_xwat_sing_geom89 & -2452854.873 & 7 & 0 \\
\hline serine_xwat_sing_geom 82 & -2452854.611 & 7 & 0 \\
\hline serine1_neutral_geom83 & -2452851.441 & 7 & 0 \\
\hline serine_xwat_sing_geom84 & -2452850.700 & 7 & 0 \\
\hline serine_xwat_sing_geom 90 & -2452848.603 & 7 & 0 \\
\hline serine1_neutral_geom85 & -2452847.540 & 7 & 0 \\
\hline serine1_neutral_geom79 & -2452843.859 & 7 & 0 \\
\hline serine_xwat_sing_geom 88 & -2452842.684 & 7 & 0 \\
\hline serine1_neutral_geom87 & -2452835.964 & 7 & 0 \\
\hline serine_xwat_sing_geom95 & -2653636.389 & 8 & 2 \\
\hline serine2_neutral_geom 92 & -2653635.745 & 8 & 2 \\
\hline serine2_neutral_geom96 & -2653635.639 & 8 & 2 \\
\hline serine2_neutral_geom94 & -2653632.966 & 8 & 2 \\
\hline serine1_neutral_geom88 & -2653628.073 & 8 & 2 \\
\hline serine2_neutral_geom95 & -2653627.428 & 8 & 2 \\
\hline
\end{tabular}




\begin{tabular}{|c|c|c|c|}
\hline serine2_neutral_geom93 & -2653627.029 & 8 & 2 \\
\hline serine2_neutral_geom97 & -2653625.914 & 8 & 2 \\
\hline serine1_neutral_geom99 & -2653625.756 & 8 & 2 \\
\hline serine_xwat_sing_geom 94 & -2653623.406 & 8 & 2 \\
\hline serine_xwat_sing_geom92 & -2653622.535 & 8 & 2 \\
\hline serine2_neutral_geom99 & -2653622.171 & 8 & 2 \\
\hline serine2_neutral_geom98 & -2653622.040 & 8 & 2 \\
\hline serine2_neutral_geom102 & -2653621.969 & 8 & 2 \\
\hline serine_xwat_sing_geom 93 & -2653620.964 & 8 & 2 \\
\hline serine1_neutral_geom89 & -2653618.653 & 8 & 2 \\
\hline serine1_neutral_geom92 & -2653618.631 & 8 & 2 \\
\hline serine1_neutral_geom95 & -2653618.042 & 8 & 2 \\
\hline serine1_neutral_geom91 & -2653617.778 & 8 & 0 \\
\hline serine2_neutral_geom100 & -2653613.133 & 8 & 2 \\
\hline serine1_neutral_geom90 & -2653612.091 & 8 & 2 \\
\hline serine_xwat_sing_geom 101 & -2653609.605 & 8 & 2 \\
\hline serine1_neutral_geom93 & -2653608.119 & 8 & 2 \\
\hline serine_xwat_sing_geom 96 & -2653607.319 & 8 & 0 \\
\hline serine1_neutral_geom94 & -2653606.445 & 8 & 2 \\
\hline serine2_neutral_geom104 & -2653604.039 & 8 & 2 \\
\hline serine2_neutral_geom103 & -2653597.650 & 8 & 2 \\
\hline serine1_neutral_geom96 & -2653595.561 & 8 & 0 \\
\hline serine_xwat_sing_geom97 & -2653589.488 & 8 & 0 \\
\hline serine_xwat_sing_geom 103 & -2653588.893 & 8 & 0 \\
\hline serine_xwat_sing_geom 102 & -2653582.511 & 8 & 0 \\
\hline serine1_neutral_geom97 & -2653579.841 & 8 & 0 \\
\hline serine_xwat_sing_geom99 & -2653578.478 & 8 & 0 \\
\hline serine1_neutral_geom98 & -2653573.360 & 8 & 0 \\
\hline serine_xwat_sing_geom104 & -2653571.961 & 8 & 0 \\
\hline serine2_neutral_geom114 & -2854385.878 & 9 & 2 \\
\hline serine_xwat_sing_geom 105 & -2854383.686 & 9 & 2 \\
\hline serine_xwat_sing_geom109 & -2854381.517 & 9 & 2 \\
\hline serine_xwat_sing_geom 107 & -2854380.793 & 9 & 2 \\
\hline serine2_neutral_geom105 & -2854375.681 & 9 & 2 \\
\hline serine1_neutral_geom101 & -2854375.048 & 9 & 2 \\
\hline serine2_neutral_geom113 & -2854372.359 & 9 & 2 \\
\hline serine1_neutral_geom102 & -2854372.201 & 9 & 2 \\
\hline serine2_neutral_geom117 & -2854371.276 & 9 & 2 \\
\hline serine1_neutral_geom104 & -2854368.138 & 9 & 2 \\
\hline
\end{tabular}




\begin{tabular}{|c|c|c|c|}
\hline serine2_neutral_geom107 & -2854367.522 & 9 & 2 \\
\hline serine2_neutral_geom112 & -2854366.919 & 9 & 2 \\
\hline serine1_neutral_geom107 & -2854364.982 & 9 & 2 \\
\hline serine2_neutral_geom110 & -2854363.338 & 9 & 2 \\
\hline serine1_neutral_geom103 & -2854363.258 & 9 & 2 \\
\hline serine2_neutral_geom106 & -2854362.506 & 9 & 2 \\
\hline serine2_neutral_geom109 & -2854362.178 & 9 & 2 \\
\hline serine2_neutral_geom108 & -2854360.976 & 9 & 2 \\
\hline serine1_neutral_geom105 & -2854356.407 & 9 & 2 \\
\hline serine_xwat_sing_geom 106 & -2854351.836 & 9 & 2 \\
\hline serine_xwat_sing_geom 108 & -2854350.949 & 9 & 2 \\
\hline serine1_neutral_geom106 & -2854350.282 & 9 & 0 \\
\hline serine1_neutral_geom111 & -2854346.635 & 9 & 2 \\
\hline serine2_neutral_geom111 & -2854342.948 & 9 & 2 \\
\hline serine2_neutral_geom116 & -2854342.028 & 9 & 2 \\
\hline serine2_neutral_geom115 & -2854340.390 & 9 & 2 \\
\hline serine1_neutral_geom113 & -2854336.168 & 9 & 0 \\
\hline serine1_neutral_geom109 & -2854336.017 & 9 & 0 \\
\hline serine1_neutral_geom112 & -2854329.371 & 9 & 0 \\
\hline serine1_neutral_geom110 & -2854325.123 & 9 & 0 \\
\hline serine1_neutral_geom108 & -2854319.365 & 9 & 0 \\
\hline serine1_neutral_geom115 & -3055128.202 & 10 & 2 \\
\hline serine2_neutral_geom118 & -3055125.746 & 10 & 2 \\
\hline serine1_neutral_geom118 & -3055124.659 & 10 & 2 \\
\hline serine2_neutral_geom119 & -3055116.862 & 10 & 2 \\
\hline serine_xwat_sing_geom 126 & -3055115.939 & 10 & 2 \\
\hline serine1_neutral_geom114 & -3055114.018 & 10 & 2 \\
\hline serine2_neutral_geom121 & -3055110.564 & 10 & 2 \\
\hline serine2_neutral_geom124 & -3055110.185 & 10 & 2 \\
\hline serine_xwat_sing_geom 129 & -3055109.844 & 10 & 2 \\
\hline serine2_neutral_geom130 & -3055107.818 & 10 & 2 \\
\hline serine_xwat_sing_geom 128 & -3055106.245 & 10 & 2 \\
\hline serine2_neutral_geom123 & -3055104.718 & 10 & 2 \\
\hline serine1_neutral_geom116 & -3055104.219 & 10 & 2 \\
\hline serine_xwat_sing_geom 122 & -3055103.694 & 10 & 2 \\
\hline serine1_neutral_geom117 & -3055102.237 & 10 & 2 \\
\hline serine2_neutral_geom 120 & -3055099.865 & 10 & 2 \\
\hline serine1_neutral_geom120 & -3055099.736 & 10 & 2 \\
\hline serine_xwat_sing_geom 124 & -3055099.102 & 10 & 2 \\
\hline
\end{tabular}




\begin{tabular}{|c|c|c|c|}
\hline serine2_neutral_geom122 & -3055098.875 & 10 & 2 \\
\hline serine2_neutral_geom127 & -3055095.739 & 10 & 2 \\
\hline serine1_neutral_geom121 & -3055091.542 & 10 & 2 \\
\hline serine1_neutral_geom119 & -3055090.386 & 10 & 2 \\
\hline serine_xwat_sing_geom 120 & -3055087.239 & 10 & 0 \\
\hline serine1_neutral_geom125 & -3055082.128 & 10 & 0 \\
\hline serine1_neutral_geom123 & -3055082.077 & 10 & 2 \\
\hline serine1_neutral_geom124 & -3055081.368 & 10 & 2 \\
\hline serine_xwat_sing_geom 123 & -3055079.954 & 10 & 0 \\
\hline serine2_neutral_geom126 & -3055079.213 & 10 & 0 \\
\hline serine_xwat_sing_geom 121 & -3055078.966 & 10 & 0 \\
\hline serine1_neutral_geom122 & -3055078.127 & 10 & 0 \\
\hline serine2_neutral_geom 125 & -3055076.645 & 10 & 0 \\
\hline serine_xwat_sing_geom 127 & -3055074.463 & 10 & 0 \\
\hline serine_xwat_sing_geom 130 & -3055072.471 & 10 & 0 \\
\hline serine2_neutral_geom129 & -3055072.276 & 10 & 0 \\
\hline serine2_neutral_geom128 & -3055069.096 & 10 & 0 \\
\hline serine1_neutral_geom126 & -3055068.056 & 10 & 0 \\
\hline serine_xwat_sing_geom 125 & -3055052.932 & 10 & 0 \\
\hline serine_xwat_sing_geom 132 & -3255871.783 & 11 & 2 \\
\hline serine_xwat_sing_geom 131 & -3255862.355 & 11 & 2 \\
\hline serine_xwat_sing_geom 133 & -3255858.826 & 11 & 2 \\
\hline serine2_neutral_geom131 & -3255854.885 & 11 & 2 \\
\hline serine_xwat_sing_geom 135 & -3255852.557 & 11 & 2 \\
\hline serine2_neutral_geom135 & -3255852.517 & 11 & 2 \\
\hline serine1_neutral_geom133 & -3255852.306 & 11 & 2 \\
\hline serine1_neutral_geom127 & -3255852.154 & 11 & 2 \\
\hline serine1_neutral_geom129 & -3255850.984 & 11 & 2 \\
\hline serine2_neutral_geom132 & -3255848.834 & 11 & 2 \\
\hline serine2_neutral_geom137 & -3255847.147 & 11 & 2 \\
\hline serine_xwat_sing_geom 137 & -3255846.508 & 11 & 2 \\
\hline serine2_neutral_geom133 & -3255843.981 & 11 & 2 \\
\hline serine2_neutral_geom134 & -3255840.755 & 11 & 2 \\
\hline serine1_neutral_geom128 & -3255840.710 & 11 & 2 \\
\hline serine1_neutral_geom139 & -3255838.151 & 11 & 2 \\
\hline serine_xwat_sing_geom 143 & -3255837.357 & 11 & 2 \\
\hline serine_xwat_sing_geom 136 & -3255835.975 & 11 & 2 \\
\hline serine1_neutral_geom131 & -3255833.572 & 11 & 0 \\
\hline serine2_neutral_geom142 & -3255833.070 & 11 & 2 \\
\hline
\end{tabular}




\begin{tabular}{|c|c|c|c|}
\hline serine_xwat_sing_geom 134 & -3255832.568 & 11 & 2 \\
\hline serine2_neutral_geom136 & -3255831.510 & 11 & 2 \\
\hline serine2_neutral_geom140 & -3255831.262 & 11 & 0 \\
\hline serine_xwat_sing_geom139 & -3255831.158 & 11 & 0 \\
\hline serine1_neutral_geom137 & -3255831.112 & 11 & 0 \\
\hline serine2_neutral_geom139 & -3255830.724 & 11 & 0 \\
\hline serine2_neutral_geom138 & -3255830.195 & 11 & 2 \\
\hline serine2_neutral_geom141 & -3255829.898 & 11 & 2 \\
\hline serine1_neutral_geom134 & -3255827.508 & 11 & 2 \\
\hline serine1_neutral_geom135 & -3255826.343 & 11 & 2 \\
\hline serine1_neutral_geom132 & -3255824.419 & 11 & 0 \\
\hline serine_xwat_sing_geom 141 & -3255821.497 & 11 & 0 \\
\hline serine_xwat_sing_geom 142 & -3255816.079 & 11 & 2 \\
\hline serine1_neutral_geom138 & -3255815.337 & 11 & 0 \\
\hline serine_xwat_sing_geom 140 & -3255813.136 & 11 & 0 \\
\hline serine1_neutral_geom130 & -3255812.986 & 11 & 0 \\
\hline serine1_neutral_geom136 & -3255802.768 & 11 & 0 \\
\hline serine2_neutral_geom143 & -3255796.164 & 11 & 0 \\
\hline serine2_neutral_geom154 & -3456619.772 & 12 & 2 \\
\hline serine1_neutral_geom144 & -3456614.745 & 12 & 2 \\
\hline serine_xwat_sing_geom 150 & -3456610.478 & 12 & 2 \\
\hline serine1_neutral_geom148 & -3456606.455 & 12 & 2 \\
\hline serine_xwat_sing_geom 144 & -3456603.477 & 12 & 0 \\
\hline serine2_neutral_geom147 & -3456601.024 & 12 & 2 \\
\hline serine2_neutral_geom152 & -3456599.063 & 12 & 2 \\
\hline serine1_neutral_geom140 & -3456595.858 & 12 & 2 \\
\hline serine2_neutral_geom149 & -3456595.174 & 12 & 2 \\
\hline serine2_neutral_geom145 & -3456592.586 & 12 & 2 \\
\hline serine1_neutral_geom145 & -3456584.393 & 12 & 2 \\
\hline serine1_neutral_geom 150 & -3456582.550 & 12 & 2 \\
\hline serine2_neutral_geom144 & -3456582.389 & 12 & 2 \\
\hline serine1_neutral_geom142 & -3456582.325 & 12 & 2 \\
\hline serine_xwat_sing_geom 155 & -3456581.203 & 12 & 2 \\
\hline serine_xwat_sing_geom145 & -3456579.817 & 12 & 2 \\
\hline serine_xwat_sing_geom 152 & -3456579.091 & 12 & 0 \\
\hline serine2_neutral_geom155 & -3456577.096 & 12 & 2 \\
\hline serine_xwat_sing_geom 151 & -3456576.102 & 12 & 0 \\
\hline serine1_neutral_geom147 & -3456575.840 & 12 & 1 \\
\hline serine2_neutral_geom148 & -3456575.094 & 12 & 2 \\
\hline
\end{tabular}




\begin{tabular}{|c|c|c|c|}
\hline serine1_neutral_geom141 & -3456572.976 & 12 & 2 \\
\hline serine2_neutral_geom150 & -3456571.955 & 12 & 2 \\
\hline serine_xwat_sing_geom149 & -3456570.517 & 12 & 2 \\
\hline serine1_neutral_geom143 & -3456570.317 & 12 & 2 \\
\hline serine2_neutral_geom153 & -3456569.270 & 12 & 2 \\
\hline serine2_neutral_geom156 & -3456569.101 & 12 & 2 \\
\hline serine2_neutral_geom 151 & -3456565.766 & 12 & 2 \\
\hline serine2_neutral_geom146 & -3456565.344 & 12 & 0 \\
\hline serine_xwat_sing_geom 148 & -3456561.156 & 12 & 0 \\
\hline serine_xwat_sing_geom 153 & -3456561.146 & 12 & 2 \\
\hline serine1_neutral_geom149 & -3456559.612 & 12 & 0 \\
\hline serine_xwat_sing_geom 154 & -3456557.288 & 12 & 1 \\
\hline serine1_neutral_geom152 & -3456553.525 & 12 & 0 \\
\hline serine_xwat_sing_geom 147 & -3456551.456 & 12 & 0 \\
\hline serine_xwat_sing_geom 146 & -3456550.826 & 12 & 2 \\
\hline serine_xwat_sing_geom 156 & -3456550.141 & 12 & 0 \\
\hline serine1_neutral_geom151 & -3456544.448 & 12 & 0 \\
\hline serine1_neutral_geom146 & -3456539.847 & 12 & 0 \\
\hline serine_xwat_sing_geom 157 & -3657356.164 & 13 & 2 \\
\hline serine1_neutral_geom163 & -3657351.283 & 13 & 2 \\
\hline serine_xwat_sing_geom 159 & -3657339.612 & 13 & 2 \\
\hline serine2_neutral_geom169 & -3657339.482 & 13 & 2 \\
\hline serine2_neutral_geom159 & -3657338.316 & 13 & 2 \\
\hline serine1_neutral_geom 153 & -3657336.066 & 13 & 2 \\
\hline serine_xwat_sing_geom164 & -3657334.345 & 13 & 1 \\
\hline serine2_neutral_geom157 & -3657333.947 & 13 & 2 \\
\hline serine1_neutral_geom155 & -3657332.971 & 13 & 1 \\
\hline serine1_neutral_geom154 & -3657332.449 & 13 & 2 \\
\hline serine_xwat_sing_geom 160 & -3657329.148 & 13 & 2 \\
\hline serine2_neutral_geom161 & -3657325.938 & 13 & 2 \\
\hline serine2_neutral_geom168 & -3657324.792 & 13 & 2 \\
\hline serine1_neutral_geom156 & -3657323.495 & 13 & 2 \\
\hline serine2_neutral_geom160 & -3657322.664 & 13 & 2 \\
\hline serine2_neutral_geom163 & -3657320.020 & 13 & 2 \\
\hline serine_xwat_sing_geom 162 & -3657319.888 & 13 & 0 \\
\hline serine2_neutral_geom162 & -3657315.847 & 13 & 1 \\
\hline serine2_neutral_geom165 & -3657313.838 & 13 & 2 \\
\hline serine2_neutral_geom167 & -3657312.126 & 13 & 0 \\
\hline serine2_neutral_geom166 & -3657306.638 & 13 & 0 \\
\hline
\end{tabular}




\begin{tabular}{|c|c|c|c|}
\hline serine1_neutral_geom165 & -3657305.366 & 13 & 0 \\
\hline serine_xwat_sing_geom 163 & -3657305.188 & 13 & 1 \\
\hline serine_xwat_sing_geom 158 & -3657304.856 & 13 & 0 \\
\hline serine_xwat_sing_geom 161 & -3657304.085 & 13 & 2 \\
\hline serine1_neutral_geom158 & -3657303.718 & 13 & 2 \\
\hline serine2_neutral_geom164 & -3657302.732 & 13 & 0 \\
\hline serine_xwat_sing_geom 165 & -3657301.257 & 13 & 2 \\
\hline serine1_neutral_geom159 & -3657297.165 & 13 & 0 \\
\hline serine1_neutral_geom160 & -3657296.346 & 13 & 0 \\
\hline serine1_neutral_geom161 & -3657294.588 & 13 & 0 \\
\hline serine_xwat_sing_geom 167 & -3657293.726 & 13 & 0 \\
\hline serine_xwat_sing_geom169 & -3657291.361 & 13 & 0 \\
\hline serine_xwat_sing_geom 168 & -3657289.166 & 13 & 0 \\
\hline serine1_neutral_geom164 & -3657288.526 & 13 & 0 \\
\hline serine1_neutral_geom157 & -3657286.622 & 13 & 2 \\
\hline serine_xwat_sing_geom166 & -3657285.069 & 13 & 0 \\
\hline serine1_neutral_geom162 & -3657283.543 & 13 & 0 \\
\hline serine2_neutral_geom170 & -3858118.391 & 14 & 2 \\
\hline serine2_neutral_geom172 & -3858114.710 & 14 & 2 \\
\hline serine1_neutral_geom173 & -3858090.886 & 14 & 2 \\
\hline serine2_neutral_geom171 & -3858088.326 & 14 & 2 \\
\hline serine2_neutral_geom175 & -3858086.015 & 14 & 2 \\
\hline serine2_neutral_geom178 & -3858083.823 & 14 & 2 \\
\hline serine2_neutral_geom 174 & -3858082.194 & 14 & 2 \\
\hline serine2_neutral_geom173 & -3858078.750 & 14 & 2 \\
\hline serine1_neutral_geom166 & -3858076.551 & 14 & 2 \\
\hline serine1_neutral_geom167 & -3858076.500 & 14 & 2 \\
\hline serine2_neutral_geom176 & -3858074.980 & 14 & 2 \\
\hline serine_xwat_sing_geom 170 & -3858072.985 & 14 & 2 \\
\hline serine1_neutral_geom168 & -3858072.132 & 14 & 2 \\
\hline serine_xwat_sing_geom 171 & -3858071.139 & 14 & 2 \\
\hline serine_xwat_sing_geom 177 & -3858069.005 & 14 & 2 \\
\hline serine2_neutral_geom181 & -3858068.552 & 14 & 2 \\
\hline serine_xwat_sing_geom 180 & -3858067.815 & 14 & 2 \\
\hline serine2_neutral_geom180 & -3858066.400 & 14 & 0 \\
\hline serine1_neutral_geom169 & -3858065.308 & 14 & 2 \\
\hline serine2_neutral_geom179 & -3858065.004 & 14 & 2 \\
\hline serine_xwat_sing_geom 172 & -3858063.100 & 14 & 0 \\
\hline serine_xwat_sing_geom 175 & -3858062.214 & 14 & 1 \\
\hline
\end{tabular}




\begin{tabular}{|c|c|c|c|}
\hline serine2_neutral_geom 182 & -3858059.311 & 14 & 2 \\
\hline serine2_neutral_geom177 & -3858058.220 & 14 & 2 \\
\hline serine_xwat_sing_geom 181 & -3858057.417 & 14 & 2 \\
\hline serine_xwat_sing_geom 174 & -3858055.114 & 14 & 2 \\
\hline serine1_neutral_geom172 & -3858053.424 & 14 & 2 \\
\hline serine_xwat_sing_geom 173 & -3858049.580 & 14 & 1 \\
\hline serine_xwat_sing_geom 178 & -3858049.397 & 14 & 2 \\
\hline serine1_neutral_geom 170 & -3858048.245 & 14 & 2 \\
\hline serine1_neutral_geom174 & -3858048.139 & 14 & 0 \\
\hline serine1_neutral_geom175 & -3858044.031 & 14 & 1 \\
\hline serine1_neutral_geom177 & -3858043.741 & 14 & 0 \\
\hline serine1_neutral_geom171 & -3858043.185 & 14 & 0 \\
\hline serine1_neutral_geom176 & -3858036.291 & 14 & 0 \\
\hline serine_xwat_sing_geom 182 & -3858033.062 & 14 & 0 \\
\hline serine1_neutral_geom178 & -3858032.058 & 14 & 0 \\
\hline serine_xwat_sing_geom 176 & -3858031.414 & 14 & 0 \\
\hline serine_xwat_sing_geom179 & -3858028.758 & 14 & 1 \\
\hline serine2_neutral_geom185 & -4058852.795 & 15 & 2 \\
\hline serine2_neutral_geom187 & -4058835.500 & 15 & 2 \\
\hline serine2_neutral_geom183 & -4058834.886 & 15 & 2 \\
\hline serine1_neutral_geom179 & -4058828.078 & 15 & 2 \\
\hline serine2_neutral_geom189 & -4058824.230 & 15 & 2 \\
\hline serine_xwat_sing_geom 183 & -4058822.624 & 15 & 1 \\
\hline serine2_neutral_geom184 & -4058818.012 & 15 & 2 \\
\hline serine2_neutral_geom186 & -4058817.901 & 15 & 2 \\
\hline serine_xwat_sing_geom 185 & -4058817.171 & 15 & 2 \\
\hline serine_xwat_sing_geom 184 & -4058816.828 & 15 & 2 \\
\hline serine1_neutral_geom180 & -4058814.004 & 15 & 2 \\
\hline serine2_neutral_geom188 & -4058811.704 & 15 & 2 \\
\hline serine2_neutral_geom192 & -4058810.895 & 15 & 2 \\
\hline serine_xwat_sing_geom 186 & -4058808.795 & 15 & 2 \\
\hline serine1_neutral_geom181 & -4058808.311 & 15 & 2 \\
\hline serine2_neutral_geom190 & -4058808.080 & 15 & 2 \\
\hline serine2_neutral_geom191 & -4058806.495 & 15 & 2 \\
\hline serine1_neutral_geom187 & -4058804.792 & 15 & 2 \\
\hline serine1_neutral_geom182 & -4058803.318 & 15 & 2 \\
\hline serine_xwat_sing_geom 187 & -4058802.208 & 15 & 2 \\
\hline serine1_neutral_geom185 & -4058801.974 & 15 & 2 \\
\hline serine_xwat_sing_geom 188 & -4058800.137 & 15 & 1 \\
\hline
\end{tabular}




\begin{tabular}{|c|c|c|c|}
\hline serine2_neutral_geom193 & -4058799.007 & 15 & 0 \\
\hline serine1_neutral_geom186 & -4058798.865 & 15 & 2 \\
\hline serine_xwat_sing_geom 191 & -4058796.157 & 15 & 2 \\
\hline serine1_neutral_geom184 & -4058788.190 & 15 & 2 \\
\hline serine1_neutral_geom183 & -4058787.503 & 15 & 1 \\
\hline serine1_neutral_geom190 & -4058786.557 & 15 & 0 \\
\hline serine_xwat_sing_geom 193 & -4058784.773 & 15 & 0 \\
\hline serine_xwat_sing_geom 190 & -4058782.644 & 15 & 0 \\
\hline serine1_neutral_geom189 & -4058780.420 & 15 & 0 \\
\hline serine_xwat_sing_geom 192 & -4058778.959 & 15 & 2 \\
\hline serine2_neutral_geom194 & -4058776.305 & 15 & 0 \\
\hline serine_xwat_sing_geom 189 & -4058774.265 & 15 & 0 \\
\hline serine_xwat_sing_geom 194 & -4058774.173 & 15 & 0 \\
\hline serine_xwat_sing_geom 195 & -4058773.109 & 15 & 0 \\
\hline serine1_neutral_geom191 & -4058772.525 & 15 & 0 \\
\hline serine1_neutral_geom188 & -4058770.237 & 15 & 2 \\
\hline serine2_neutral_geom195 & -4058754.985 & 15 & 2 \\
\hline serine_xwat_sing_geom 204 & -4259590.651 & 16 & 2 \\
\hline serine1_neutral_geom192 & -4259581.798 & 16 & 2 \\
\hline serine1_neutral_geom194 & -4259581.178 & 16 & 2 \\
\hline serine2_neutral_geom196 & -4259573.950 & 16 & 2 \\
\hline serine_xwat_sing_geom 200 & -4259573.270 & 16 & 2 \\
\hline serine2_neutral_geom201 & -4259571.207 & 16 & 2 \\
\hline serine_xwat_sing_geom 202 & -4259570.985 & 16 & 1 \\
\hline serine2_neutral_geom200 & -4259567.140 & 16 & 2 \\
\hline serine2_neutral_geom204 & -4259566.828 & 16 & 2 \\
\hline serine2_neutral_geom199 & -4259565.932 & 16 & 2 \\
\hline serine2_neutral_geom197 & -4259565.516 & 16 & 2 \\
\hline serine_xwat_sing_geom196 & -4259564.391 & 16 & 2 \\
\hline serine2_neutral_geom198 & -4259562.350 & 16 & 2 \\
\hline serine2_neutral_geom205 & -4259558.932 & 16 & 2 \\
\hline serine2_neutral_geom202 & -4259556.500 & 16 & 2 \\
\hline serine_xwat_sing_geom197 & -4259556.188 & 16 & 2 \\
\hline serine2_neutral_geom208 & -4259549.469 & 16 & 2 \\
\hline serine1_neutral_geom196 & -4259549.114 & 16 & 2 \\
\hline serine2_neutral_geom203 & -4259549.056 & 16 & 2 \\
\hline serine2_neutral_geom207 & -4259545.383 & 16 & 2 \\
\hline serine2_neutral_geom206 & -4259544.714 & 16 & 2 \\
\hline serine1_neutral_geom195 & -4259544.648 & 16 & 2 \\
\hline
\end{tabular}




\begin{tabular}{|c|c|c|c|}
\hline serine1_neutral_geom198 & -4259544.526 & 16 & 2 \\
\hline serine_xwat_sing_geom 203 & -4259542.433 & 16 & 1 \\
\hline serine_xwat_sing_geom198 & -4259540.244 & 16 & 2 \\
\hline serine1_neutral_geom193 & -4259532.791 & 16 & 2 \\
\hline serine_xwat_sing_geom 205 & -4259529.342 & 16 & 0 \\
\hline serine1_neutral_geom 200 & -4259522.809 & 16 & 1 \\
\hline serine_xwat_sing_geom 201 & -4259520.384 & 16 & 0 \\
\hline serine_xwat_sing_geom 208 & -4259517.666 & 16 & 0 \\
\hline serine1_neutral_geom202 & -4259516.298 & 16 & 0 \\
\hline serine1_neutral_geom204 & -4259513.006 & 16 & 0 \\
\hline serine_xwat_sing_geom 207 & -4259512.301 & 16 & 0 \\
\hline serine1_neutral_geom197 & -4259511.425 & 16 & 0 \\
\hline serine1_neutral_geom203 & -4259509.394 & 16 & 0 \\
\hline serine_xwat_sing_geom 206 & -4259507.099 & 16 & 0 \\
\hline serine1_neutral_geom199 & -4259500.841 & 16 & 1 \\
\hline serine1_neutral_geom201 & -4259495.623 & 16 & 0 \\
\hline serine2_neutral_geom209 & -4460315.492 & 17 & 2 \\
\hline serine_xwat_sing_geom 220 & -4460311.539 & 17 & 2 \\
\hline serine2_neutral_geom210 & -4460310.180 & 17 & 2 \\
\hline serine1_neutral_geom207 & -4460309.397 & 17 & 2 \\
\hline serine1_neutral_geom205 & -4460300.353 & 17 & 2 \\
\hline serine1_neutral_geom 210 & -4460298.555 & 17 & 2 \\
\hline serine1_neutral_geom206 & -4460298.202 & 17 & 2 \\
\hline serine2_neutral_geom215 & -4460296.683 & 17 & 2 \\
\hline serine_xwat_sing_geom 215 & -4460296.447 & 17 & 2 \\
\hline serine_xwat_sing_geom 216 & -4460296.259 & 17 & 2 \\
\hline serine_xwat_sing_geom 210 & -4460295.277 & 17 & 2 \\
\hline serine2_neutral_geom219 & -4460294.021 & 17 & 2 \\
\hline serine_xwat_sing_geom 217 & -4460293.795 & 17 & 1 \\
\hline serine2_neutral_geom218 & -4460293.514 & 17 & 2 \\
\hline serine_xwat_sing_geom 209 & -4460293.110 & 17 & 2 \\
\hline serine1_neutral_geom208 & -4460291.950 & 17 & 2 \\
\hline serine_xwat_sing_geom 212 & -4460290.665 & 17 & 2 \\
\hline serine2_neutral_geom212 & -4460290.626 & 17 & 2 \\
\hline serine2_neutral_geom214 & -4460285.972 & 17 & 2 \\
\hline serine2_neutral_geom213 & -4460282.530 & 17 & 2 \\
\hline serine_xwat_sing_geom 211 & -4460281.079 & 17 & 0 \\
\hline serine2_neutral_geom216 & -4460279.726 & 17 & 2 \\
\hline serine1_neutral_geom 211 & -4460279.124 & 17 & 0 \\
\hline
\end{tabular}




\begin{tabular}{|c|c|c|c|}
\hline serine_xwat_sing_geom 221 & -4460276.721 & 17 & 0 \\
\hline serine2_neutral_geom 217 & -4460276.707 & 17 & 2 \\
\hline serine2_neutral_geom 220 & -4460270.419 & 17 & 2 \\
\hline serine1_neutral_geom209 & -4460267.744 & 17 & 2 \\
\hline serine1_neutral_geom 213 & -4460266.901 & 17 & 0 \\
\hline serine_xwat_sing_geom 213 & -4460266.380 & 17 & 0 \\
\hline serine_xwat_sing_geom 218 & -4460264.683 & 17 & 0 \\
\hline serine2_neutral_geom 221 & -4460256.534 & 17 & 2 \\
\hline serine1_neutral_geom 214 & -4460250.404 & 17 & 0 \\
\hline serine1_neutral_geom 212 & -4460246.267 & 17 & 2 \\
\hline serine_xwat_sing_geom 214 & -4460244.393 & 17 & 1 \\
\hline serine_xwat_sing_geom 219 & -4460243.905 & 17 & 0 \\
\hline serine1_neutral_geom 217 & -4460242.887 & 17 & 0 \\
\hline serine1_neutral_geom 216 & -4460235.007 & 17 & 0 \\
\hline serine2_neutral_geom 222 & -4661072.422 & 18 & 2 \\
\hline serine2_neutral_geom224 & -4661069.474 & 18 & 2 \\
\hline serine_xwat_sing_geom 222 & -4661061.164 & 18 & 2 \\
\hline serine1_neutral_geom 230 & -4661056.175 & 18 & 2 \\
\hline serine2_neutral_geom229 & -4661054.418 & 18 & 2 \\
\hline serine2_neutral_geom226 & -4661053.886 & 18 & 2 \\
\hline serine2_neutral_geom223 & -4661048.441 & 18 & 2 \\
\hline serine_xwat_sing_geom 223 & -4661044.715 & 18 & 2 \\
\hline serine2_neutral_geom 227 & -4661039.852 & 18 & 2 \\
\hline serine_xwat_sing_geom 227 & -4661039.245 & 18 & 2 \\
\hline serine_xwat_sing_geom 228 & -4661037.393 & 18 & 2 \\
\hline serine2_neutral_geom225 & -4661036.563 & 18 & 2 \\
\hline serine2_neutral_geom 230 & -4661036.488 & 18 & 2 \\
\hline serine_xwat_sing_geom 226 & -4661035.699 & 18 & 0 \\
\hline serine2_neutral_geom233 & -4661034.667 & 18 & 2 \\
\hline serine2_neutral_geom232 & -4661032.613 & 18 & 2 \\
\hline serine1_neutral_geom 222 & -4661031.169 & 18 & 2 \\
\hline serine1_neutral_geom 218 & -4661030.809 & 18 & 2 \\
\hline serine_xwat_sing_geom 231 & -4661030.683 & 18 & 2 \\
\hline serine_xwat_sing_geom 225 & -4661030.556 & 18 & 2 \\
\hline serine1_neutral_geom 228 & -4661029.203 & 18 & 0 \\
\hline serine1_neutral_geom219 & -4661028.511 & 18 & 2 \\
\hline serine_xwat_sing_geom 224 & -4661022.511 & 18 & 2 \\
\hline serine2_neutral_geom228 & -4661020.649 & 18 & 2 \\
\hline serine1_neutral_geom 220 & -4661019.798 & 18 & 2 \\
\hline
\end{tabular}




\begin{tabular}{|c|c|c|c|}
\hline serine2_neutral_geom234 & -4661017.603 & 18 & 2 \\
\hline serine_xwat_sing_geom 230 & -4661012.643 & 18 & 2 \\
\hline serine1_neutral_geom 221 & -4661007.974 & 18 & 2 \\
\hline serine1_neutral_geom224 & -4661004.135 & 18 & 1 \\
\hline serine_xwat_sing_geom 234 & -4661003.751 & 18 & 0 \\
\hline serine_xwat_sing_geom 233 & -4661000.969 & 18 & 2 \\
\hline serine1_neutral_geom223 & -4660998.057 & 18 & 0 \\
\hline serine_xwat_sing_geom 229 & -4660994.815 & 18 & 0 \\
\hline serine1_neutral_geom229 & -4660994.562 & 18 & 0 \\
\hline serine_xwat_sing_geom 232 & -4660985.161 & 18 & 0 \\
\hline serine1_neutral_geom227 & -4660974.831 & 18 & 0 \\
\hline serine2_neutral_geom239 & -4861808.144 & 19 & 2 \\
\hline serine_xwat_sing_geom 235 & -4861796.822 & 19 & 2 \\
\hline serine2_neutral_geom235 & -4861793.797 & 19 & 2 \\
\hline serine1_neutral_geom 231 & -4861792.553 & 19 & 2 \\
\hline serine2_neutral_geom237 & -4861787.642 & 19 & 2 \\
\hline serine2_neutral_geom245 & -4861786.908 & 19 & 2 \\
\hline serine_xwat_sing_geom 239 & -4861783.104 & 19 & 2 \\
\hline serine1_neutral_geom 238 & -4861780.526 & 19 & 2 \\
\hline serine1_neutral_geom233 & -4861780.276 & 19 & 2 \\
\hline serine2_neutral_geom242 & -4861779.760 & 19 & 2 \\
\hline serine2_neutral_geom246 & -4861779.653 & 19 & 2 \\
\hline serine1_neutral_geom 232 & -4861779.258 & 19 & 2 \\
\hline serine_xwat_sing_geom 246 & -4861778.471 & 19 & 2 \\
\hline serine1_neutral_geom243 & -4861778.464 & 19 & 2 \\
\hline serine2_neutral_geom244 & -4861777.754 & 19 & 2 \\
\hline serine2_neutral_geom236 & -4861776.994 & 19 & 2 \\
\hline serine1_neutral_geom 236 & -4861776.569 & 19 & 1 \\
\hline serine_xwat_sing_geom 241 & -4861776.509 & 19 & 2 \\
\hline serine_xwat_sing_geom 242 & -4861774.300 & 19 & 0 \\
\hline serine2_neutral_geom 243 & -4861772.116 & 19 & 2 \\
\hline serine2_neutral_geom241 & -4861769.093 & 19 & 2 \\
\hline serine_xwat_sing_geom 244 & -4861763.731 & 19 & 2 \\
\hline serine_xwat_sing_geom 238 & -4861763.716 & 19 & 2 \\
\hline serine1_neutral_geom234 & -4861760.288 & 19 & 2 \\
\hline serine1_neutral_geom237 & -4861757.460 & 19 & 0 \\
\hline serine_xwat_sing_geom 243 & -4861756.761 & 19 & 0 \\
\hline serine1_neutral_geom235 & -4861755.846 & 19 & 2 \\
\hline serine2_neutral_geom247 & -4861748.644 & 19 & 2 \\
\hline
\end{tabular}




\begin{tabular}{|c|c|c|c|}
\hline serine_xwat_sing_geom 236 & -4861744.350 & 19 & 0 \\
\hline serine_xwat_sing_geom 237 & -4861744.341 & 19 & 0 \\
\hline serine1_neutral_geom 240 & -4861744.001 & 19 & 1 \\
\hline serine_xwat_sing_geom 240 & -4861742.749 & 19 & 0 \\
\hline serine_xwat_sing_geom 245 & -4861739.003 & 19 & 0 \\
\hline serine1_neutral_geom239 & -4861721.975 & 19 & 0 \\
\hline serine1_neutral_geom241 & -4861709.745 & 19 & 0 \\
\hline serine1_neutral_geom255 & -5062579.111 & 20 & 2 \\
\hline serine1_neutral_geom244 & -5062571.874 & 20 & 2 \\
\hline serine2_neutral_geom249 & -5062568.230 & 20 & 2 \\
\hline serine2_neutral_geom248 & -5062564.935 & 20 & 2 \\
\hline serine1_neutral_geom248 & -5062553.251 & 20 & 2 \\
\hline serine1_neutral_geom245 & -5062552.639 & 20 & 2 \\
\hline serine2_neutral_geom 251 & -5062542.330 & 20 & 2 \\
\hline serine1_neutral_geom246 & -5062540.482 & 20 & 2 \\
\hline serine2_neutral_geom256 & -5062535.968 & 20 & 2 \\
\hline serine_xwat_sing_geom 260 & -5062535.554 & 20 & 2 \\
\hline serine2_neutral_geom254 & -5062532.522 & 20 & 2 \\
\hline serine1_neutral_geom247 & -5062532.366 & 20 & 2 \\
\hline serine2_neutral_geom 253 & -5062529.123 & 20 & 2 \\
\hline serine2_neutral_geom259 & -5062525.327 & 20 & 2 \\
\hline serine2_neutral_geom 257 & -5062523.626 & 20 & 2 \\
\hline serine_xwat_sing_geom 250 & -5062519.823 & 20 & 2 \\
\hline serine_xwat_sing_geom 256 & -5062519.454 & 20 & 2 \\
\hline serine1_neutral_geom251 & -5062518.425 & 20 & 2 \\
\hline serine1_neutral_geom249 & -5062517.140 & 20 & 2 \\
\hline serine2_neutral_geom 250 & -5062517.102 & 20 & 2 \\
\hline serine_xwat_sing_geom 249 & -5062516.183 & 20 & 2 \\
\hline serine1_neutral_geom253 & -5062514.707 & 20 & 2 \\
\hline serine_xwat_sing_geom 255 & -5062514.364 & 20 & 1 \\
\hline serine_xwat_sing_geom 253 & -5062513.136 & 20 & 0 \\
\hline serine1_neutral_geom250 & -5062511.638 & 20 & 2 \\
\hline serine2_neutral_geom258 & -5062510.879 & 20 & 2 \\
\hline serine2_neutral_geom255 & -5062507.338 & 20 & 2 \\
\hline serine2_neutral_geom 252 & -5062505.372 & 20 & 2 \\
\hline serine1_neutral_geom 252 & -5062504.421 & 20 & 1 \\
\hline serine_xwat_sing_geom 258 & -5062504.084 & 20 & 0 \\
\hline serine2_neutral_geom260 & -5062503.479 & 20 & 2 \\
\hline serine1_neutral_geom 254 & -5062503.228 & 20 & 0 \\
\hline
\end{tabular}




\begin{tabular}{|l|l|l|l|}
\hline serine_xwat_sing_geom259 & -5062501.818 & 20 & 2 \\
\hline serine_xwat_sing_geom252 & -5062498.551 & 20 & 2 \\
\hline serine_xwat_sing_geom251 & -5062495.151 & 20 & 2 \\
\hline serine_xwat_sing_geom257 & -5062486.563 & 20 & 0 \\
\hline serine1_neutral_geom256 & -5062480.433 & 20 & 0 \\
\hline serine_xwat_sing_geom254 & -5062480.227 & 20 & 2 \\
\hline
\end{tabular}


Table S6 - Protonated serine with free energy and number of waters

\begin{tabular}{|c|c|c|}
\hline File Name & Free Energy [kJ/mol] & Number of waters \\
\hline prot_serine_sing_geom1 & -1249382.14 & 1 \\
\hline prot_serine_sing_geom 2 & -1249382.12 & 1 \\
\hline prot_serine_sing_geom7 & -1249380.99 & 1 \\
\hline prot_serine_sing_geom8 & -1249380.88 & 1 \\
\hline prot_serine_sing_geom3 & -1249376.81 & 1 \\
\hline prot_serine_sing_geom6 & -1249372.82 & 1 \\
\hline prot_serine_sing_geom4 & -1249372.80 & 1 \\
\hline prot_serine_sing_geom5 & -1249372.71 & 1 \\
\hline prot_serine_sing_geom 11 & -1249369.31 & 1 \\
\hline prot_serine_sing_geom9 & -1249364.92 & 1 \\
\hline prot_serine_sing_geom 10 & -1249354.24 & 1 \\
\hline prot_serine_sing_geom 13 & -1249330.20 & 1 \\
\hline prot_serine_sing_geom 12 & -1249328.51 & 1 \\
\hline prot_serine_sing_geom 18 & -1450146.91 & 2 \\
\hline prot_serine_sing_geom19 & -1450146.65 & 2 \\
\hline prot_serine_sing_geom 20 & -1450140.45 & 2 \\
\hline prot_serine_sing_geom 25 & -1450140.12 & 2 \\
\hline prot_serine_sing_geom14 & -1450139.75 & 2 \\
\hline prot_serine_sing_geom 22 & -1450139.38 & 2 \\
\hline prot_serine_sing_geom 15 & -1450139.14 & 2 \\
\hline prot_serine_sing_geom 26 & -1450138.77 & 2 \\
\hline prot_serine_sing_geom 23 & -1450138.21 & 2 \\
\hline prot_serine_sing_geom 17 & -1450134.19 & 2 \\
\hline prot_serine_sing_geom 16 & -1450133.96 & 2 \\
\hline prot_serine_sing_geom 24 & -1450133.78 & 2 \\
\hline prot_serine_sing_geom 21 & -1450124.59 & 2 \\
\hline prot_serine_sing_geom 28 & -1650900.46 & 3 \\
\hline prot_serine_sing_geom 37 & -1650899.36 & 3 \\
\hline prot_serine_sing_geom 29 & -1650898.43 & 3 \\
\hline prot_serine_sing_geom 36 & -1650898.28 & 3 \\
\hline prot_serine_sing_geom 39 & -1650896.50 & 3 \\
\hline prot_serine_sing_geom 33 & -1650891.79 & 3 \\
\hline prot_serine_sing_geom 38 & -1650890.75 & 3 \\
\hline prot_serine_sing_geom 27 & -1650890.54 & 3 \\
\hline prot_serine_sing_geom 32 & -1650888.87 & 3 \\
\hline prot_serine_sing_geom 35 & -1650888.08 & 3 \\
\hline prot_serine_sing_geom 31 & -1650887.92 & 3 \\
\hline
\end{tabular}




\begin{tabular}{|c|c|c|}
\hline prot_serine_sing_geom 30 & -1650887.72 & 3 \\
\hline prot_serine_sing_geom 34 & -1650877.02 & 3 \\
\hline prot_serine_sing_geom 40 & -1851645.87 & 4 \\
\hline prot_serine_sing_geom 42 & -1851645.07 & 4 \\
\hline prot_serine_sing_geom 43 & -1851644.92 & 4 \\
\hline prot_serine_sing_geom 41 & -1851644.89 & 4 \\
\hline prot_serine_sing_geom 44 & -1851644.68 & 4 \\
\hline prot_serine_sing_geom52 & -1851643.73 & 4 \\
\hline prot_serine_sing_geom 50 & -1851641.86 & 4 \\
\hline prot_serine_sing_geom 47 & -1851640.29 & 4 \\
\hline prot_serine_sing_geom51 & -1851639.10 & 4 \\
\hline prot_serine_sing_geom 48 & -1851638.88 & 4 \\
\hline prot_serine_sing_geom 46 & -1851638.12 & 4 \\
\hline prot_serine_sing_geom49 & -1851637.32 & 4 \\
\hline prot_serine_sing_geom 45 & -1851633.34 & 4 \\
\hline prot_serine_sing_geom56 & -2052395.84 & 5 \\
\hline prot_serine_sing_geom64 & -2052395.77 & 5 \\
\hline prot_serine_sing_geom61 & -2052392.75 & 5 \\
\hline prot_serine_sing_geom55 & -2052392.14 & 5 \\
\hline prot_serine_sing_geom65 & -2052387.91 & 5 \\
\hline prot_serine_sing_geom57 & -2052386.12 & 5 \\
\hline prot_serine_sing_geom63 & -2052385.15 & 5 \\
\hline prot_serine_sing_geom62 & -2052384.52 & 5 \\
\hline prot_serine_sing_geom53 & -2052383.53 & 5 \\
\hline prot_serine_sing_geom54 & -2052383.40 & 5 \\
\hline prot_serine_sing_geom59 & -2052383.05 & 5 \\
\hline prot_serine_sing_geom 58 & -2052377.97 & 5 \\
\hline prot_serine_sing_geom60 & -2052376.70 & 5 \\
\hline prot_serine_sing_geom77 & -2253147.10 & 6 \\
\hline prot_serine_sing_geom70 & -2253146.75 & 6 \\
\hline prot_serine_sing_geom66 & -2253146.72 & 6 \\
\hline prot_serine_sing_geom72 & -2253137.13 & 6 \\
\hline prot_serine_sing_geom68 & -2253135.82 & 6 \\
\hline prot_serine_sing_geom71 & -2253133.59 & 6 \\
\hline prot_serine_sing_geom74 & -2253130.11 & 6 \\
\hline prot_serine_sing_geom67 & -2253126.64 & 6 \\
\hline prot_serine_sing_geom75 & -2253124.08 & 6 \\
\hline prot_serine_sing_geom73 & -2253123.99 & 6 \\
\hline prot_serine_sing_geom69 & -2253123.68 & 6 \\
\hline
\end{tabular}




\begin{tabular}{|c|c|c|}
\hline prot_serine_sing_geom 76 & -2253123.64 & 6 \\
\hline prot_serine_sing_geom 78 & -2253116.60 & 6 \\
\hline prot_serine_sing_geom 80 & -2453900.11 & 7 \\
\hline prot_serine_sing_geom 82 & -2453889.53 & 7 \\
\hline prot_serine_sing_geom79 & -2453888.12 & 7 \\
\hline prot_serine_sing_geom 89 & -2453883.88 & 7 \\
\hline prot_serine_sing_geom 81 & -2453879.82 & 7 \\
\hline prot_serine_sing_geom 84 & -2453878.27 & 7 \\
\hline prot_serine_sing_geom 87 & -2453874.06 & 7 \\
\hline prot_serine_sing_geom 90 & -2453871.85 & 7 \\
\hline prot_serine_sing_geom 88 & -2453871.75 & 7 \\
\hline prot_serine_sing_geom85 & -2453871.52 & 7 \\
\hline prot_serine_sing_geom 83 & -2453869.43 & 7 \\
\hline prot_serine_sing_geom 91 & -2453868.17 & 7 \\
\hline prot_serine_sing_geom 86 & -2453861.10 & 7 \\
\hline prot_serine_sing_geom94 & -2654630.62 & 8 \\
\hline prot_serine_sing_geom 104 & -2654630.19 & 8 \\
\hline prot_serine_sing_geom 92 & -2654629.50 & 8 \\
\hline prot_serine_sing_geom97 & -2654629.33 & 8 \\
\hline prot_serine_sing_geom99 & -2654627.45 & 8 \\
\hline prot_serine_sing_geom98 & -2654625.85 & 8 \\
\hline prot_serine_sing_geom 101 & -2654624.80 & 8 \\
\hline prot_serine_sing_geom95 & -2654624.31 & 8 \\
\hline prot_serine_sing_geom 93 & -2654623.57 & 8 \\
\hline prot_serine_sing_geom96 & -2654621.25 & 8 \\
\hline prot_serine_sing_geom 102 & -2654618.34 & 8 \\
\hline prot_serine_sing_geom 100 & -2654617.38 & 8 \\
\hline prot_serine_sing_geom 103 & -2654611.77 & 8 \\
\hline prot_serine_sing_geom 105 & -2855382.70 & 9 \\
\hline prot_serine_sing_geom 111 & -2855378.16 & 9 \\
\hline prot_serine_sing_geom 106 & -2855377.60 & 9 \\
\hline prot_serine_sing_geom 116 & -2855375.66 & 9 \\
\hline prot_serine_sing_geom 110 & -2855374.33 & 9 \\
\hline prot_serine_sing_geom 112 & -2855373.83 & 9 \\
\hline prot_serine_sing_geom 109 & -2855373.74 & 9 \\
\hline prot_serine_sing_geom 108 & -2855372.35 & 9 \\
\hline prot_serine_sing_geom 113 & -2855366.51 & 9 \\
\hline prot_serine_sing_geom 114 & -2855364.16 & 9 \\
\hline prot_serine_sing_geom 117 & -2855363.84 & 9 \\
\hline
\end{tabular}




\begin{tabular}{|c|c|c|}
\hline prot_serine_sing_geom 107 & -2855352.48 & 9 \\
\hline prot_serine_sing_geom 115 & -2855348.82 & 9 \\
\hline prot_serine_sing_geom 120 & -3056140.67 & 10 \\
\hline prot_serine_sing_geom 118 & -3056131.65 & 10 \\
\hline prot_serine_sing_geom 122 & -3056130.51 & 10 \\
\hline prot_serine_sing_geom 127 & -3056126.57 & 10 \\
\hline prot_serine_sing_geom 123 & -3056125.42 & 10 \\
\hline prot_serine_sing_geom 119 & -3056121.01 & 10 \\
\hline prot_serine_sing_geom 121 & -3056120.14 & 10 \\
\hline prot_serine_sing_geom 130 & -3056113.27 & 10 \\
\hline prot_serine_sing_geom 125 & -3056112.73 & 10 \\
\hline prot_serine_sing_geom 128 & -3056107.28 & 10 \\
\hline prot_serine_sing_geom 124 & -3056106.80 & 10 \\
\hline prot_serine_sing_geom 129 & -3056106.24 & 10 \\
\hline prot_serine_sing_geom 126 & -3056106.20 & 10 \\
\hline prot_serine_sing_geom 131 & -3256887.98 & 11 \\
\hline prot_serine_sing_geom 141 & -3256886.91 & 11 \\
\hline prot_serine_sing_geom 143 & -3256874.87 & 11 \\
\hline prot_serine_sing_geom 132 & -3256873.22 & 11 \\
\hline prot_serine_sing_geom 134 & -3256872.82 & 11 \\
\hline prot_serine_sing_geom 135 & -3256863.04 & 11 \\
\hline prot_serine_sing_geom 142 & -3256862.30 & 11 \\
\hline prot_serine_sing_geom 133 & -3256862.13 & 11 \\
\hline prot_serine_sing_geom 137 & -3256862.06 & 11 \\
\hline prot_serine_sing_geom 139 & -3256858.13 & 11 \\
\hline prot_serine_sing_geom 140 & -3256856.22 & 11 \\
\hline prot_serine_sing_geom 136 & -3256852.98 & 11 \\
\hline prot_serine_sing_geom 138 & -3256844.01 & 11 \\
\hline prot_serine_sing_geom 146 & -3457614.48 & 12 \\
\hline prot_serine_sing_geom 145 & -3457614.25 & 12 \\
\hline prot_serine_sing_geom 156 & -3457612.56 & 12 \\
\hline prot_serine_sing_geom 144 & -3457605.20 & 12 \\
\hline prot_serine_sing_geom 150 & -3457602.24 & 12 \\
\hline prot_serine_sing_geom 152 & -3457599.93 & 12 \\
\hline prot_serine_sing_geom 147 & -3457598.83 & 12 \\
\hline prot_serine_sing_geom 154 & -3457598.00 & 12 \\
\hline prot_serine_sing_geom 155 & -3457595.40 & 12 \\
\hline prot_serine_sing_geom 148 & -3457592.75 & 12 \\
\hline prot_serine_sing_geom 149 & -3457592.45 & 12 \\
\hline
\end{tabular}




\begin{tabular}{|c|c|c|}
\hline prot_serine_sing_geom 153 & -3457591.65 & 12 \\
\hline prot_serine_sing_geom 151 & -3457579.23 & 12 \\
\hline prot_serine_sing_geom 157 & -3658362.58 & 13 \\
\hline prot_serine_sing_geom 158 & -3658357.18 & 13 \\
\hline prot_serine_sing_geom 168 & -3658350.84 & 13 \\
\hline prot_serine_sing_geom 159 & -3658349.19 & 13 \\
\hline prot_serine_sing_geom 169 & -3658347.58 & 13 \\
\hline prot_serine_sing_geom 167 & -3658342.55 & 13 \\
\hline prot_serine_sing_geom 164 & -3658341.03 & 13 \\
\hline prot_serine_sing_geom 163 & -3658335.57 & 13 \\
\hline prot_serine_sing_geom 165 & -3658333.92 & 13 \\
\hline prot_serine_sing_geom 161 & -3658333.42 & 13 \\
\hline prot_serine_sing_geom 160 & -3658333.25 & 13 \\
\hline prot_serine_sing_geom 162 & -3658332.88 & 13 \\
\hline prot_serine_sing_geom 166 & -3658330.93 & 13 \\
\hline prot_serine_sing_geom 170 & -3859108.29 & 14 \\
\hline prot_serine_sing_geom 177 & -3859104.57 & 14 \\
\hline prot_serine_sing_geom 171 & -3859100.99 & 14 \\
\hline prot_serine_sing_geom 172 & -3859100.45 & 14 \\
\hline prot_serine_sing_geom 174 & -3859096.22 & 14 \\
\hline prot_serine_sing_geom 176 & -3859095.29 & 14 \\
\hline prot_serine_sing_geom 173 & -3859087.31 & 14 \\
\hline prot_serine_sing_geom 175 & -3859086.23 & 14 \\
\hline prot_serine_sing_geom 181 & -3859078.28 & 14 \\
\hline prot_serine_sing_geom 178 & -3859076.18 & 14 \\
\hline prot_serine_sing_geom 179 & -3859076.15 & 14 \\
\hline prot_serine_sing_geom 180 & -3859075.78 & 14 \\
\hline prot_serine_sing_geom 182 & -3859067.23 & 14 \\
\hline prot_serine_sing_geom 183 & -4059856.67 & 15 \\
\hline prot_serine_sing_geom 186 & -4059855.07 & 15 \\
\hline prot_serine_sing_geom 184 & -4059851.52 & 15 \\
\hline prot_serine_sing_geom 191 & -4059845.52 & 15 \\
\hline prot_serine_sing_geom 192 & -4059845.03 & 15 \\
\hline prot_serine_sing_geom 185 & -4059838.82 & 15 \\
\hline prot_serine_sing_geom 190 & -4059837.82 & 15 \\
\hline prot_serine_sing_geom 188 & -4059831.79 & 15 \\
\hline prot_serine_sing_geom 189 & -4059827.43 & 15 \\
\hline prot_serine_sing_geom 195 & -4059826.94 & 15 \\
\hline prot_serine_sing_geom 194 & -4059825.99 & 15 \\
\hline
\end{tabular}




\begin{tabular}{|c|c|c|}
\hline prot_serine_sing_geom 187 & -4059822.13 & 15 \\
\hline prot_serine_sing_geom 193 & -4059812.28 & 15 \\
\hline prot_serine_sing_geom 203 & -4260591.43 & 16 \\
\hline prot_serine_sing_geom 208 & -4260589.18 & 16 \\
\hline prot_serine_sing_geom 200 & -4260587.13 & 16 \\
\hline prot_serine_sing_geom 197 & -4260583.64 & 16 \\
\hline prot_serine_sing_geom 198 & -4260576.68 & 16 \\
\hline prot_serine_sing_geom 207 & -4260576.45 & 16 \\
\hline prot_serine_sing_geom 201 & -4260571.94 & 16 \\
\hline prot_serine_sing_geom 205 & -4260571.48 & 16 \\
\hline prot_serine_sing_geom 202 & -4260569.44 & 16 \\
\hline prot_serine_sing_geom 196 & -4260567.58 & 16 \\
\hline prot_serine_sing_geom 204 & -4260566.46 & 16 \\
\hline prot_serine_sing_geom 199 & -4260559.32 & 16 \\
\hline prot_serine_sing_geom 206 & -4260542.16 & 16 \\
\hline prot_serine_sing_geom 209 & -4461340.59 & 17 \\
\hline prot_serine_sing_geom 210 & -4461332.67 & 17 \\
\hline prot_serine_sing_geom 220 & -4461331.08 & 17 \\
\hline prot_serine_sing_geom 215 & -4461323.59 & 17 \\
\hline prot_serine_sing_geom 221 & -4461322.95 & 17 \\
\hline prot_serine_sing_geom 213 & -4461318.16 & 17 \\
\hline prot_serine_sing_geom 218 & -4461314.55 & 17 \\
\hline prot_serine_sing_geom 212 & -4461313.03 & 17 \\
\hline prot_serine_sing_geom 216 & -4461311.96 & 17 \\
\hline prot_serine_sing_geom 214 & -4461310.93 & 17 \\
\hline prot_serine_sing_geom 211 & -4461307.26 & 17 \\
\hline prot_serine_sing_geom 219 & -4461306.87 & 17 \\
\hline prot_serine_sing_geom 217 & -4461303.21 & 17 \\
\hline prot_serine_sing_geom 228 & -4662096.76 & 18 \\
\hline prot_serine_sing_geom 223 & -4662092.34 & 18 \\
\hline prot_serine_sing_geom 222 & -4662088.35 & 18 \\
\hline prot_serine_sing_geom 225 & -4662088.22 & 18 \\
\hline prot_serine_sing_geom 224 & -4662082.29 & 18 \\
\hline prot_serine_sing_geom 234 & -4662076.35 & 18 \\
\hline prot_serine_sing_geom 233 & -4662073.00 & 18 \\
\hline prot_serine_sing_geom 226 & -4662067.60 & 18 \\
\hline prot_serine_sing_geom 227 & -4662066.67 & 18 \\
\hline prot_serine_sing_geom 229 & -4662055.13 & 18 \\
\hline prot_serine_sing_geom 231 & -4662050.35 & 18 \\
\hline
\end{tabular}




\begin{tabular}{|l|l|l|}
\hline prot_serine_sing_geom230 & -4662044.78 & 18 \\
\hline prot_serine_sing_geom232 & -4662036.04 & 18 \\
\hline prot_serine_sing_geom235 & -4862850.67 & 19 \\
\hline prot_serine_sing_geom238 & -4862838.49 & 19 \\
\hline prot_serine_sing_geom239 & -4862820.56 & 19 \\
\hline prot_serine_sing_geom246 & -4862818.25 & 19 \\
\hline prot_serine_sing_geom236 & -4862811.09 & 19 \\
\hline prot_serine_sing_geom241 & -4862809.41 & 19 \\
\hline prot_serine_sing_geom237 & -4862797.46 & 19 \\
\hline prot_serine_sing_geom242 & -4862795.31 & 19 \\
\hline prot_serine_sing_geom245 & -4862792.34 & 19 \\
\hline prot_serine_sing_geom240 & -4862789.66 & 19 \\
\hline prot_serine_sing_geom244 & -4862782.10 & 19 \\
\hline prot_serine_sing_geom243 & -4862779.94 & 19 \\
\hline prot_serine_sing_geom247 & -4862772.46 & 19 \\
\hline prot_serine_sing_geom248 & -5063589.18 & 20 \\
\hline prot_serine_sing_geom259 & -5063550.12 & 20 \\
\hline prot_serine_sing_geom257 & -5063549.04 & 20 \\
\hline prot_serine_sing_geom250 & -5063547.23 & 20 \\
\hline prot_serine_sing_geom252 & -5063546.37 & 20 \\
\hline prot_serine_sing_geom254 & -5063544.04 & 20 \\
\hline prot_serine_sing_geom256 & -5063543.61 & 20 \\
\hline prot_serine_sing_geom249 & -5063537.76 & 20 \\
\hline prot_serine_sing_geom260 & -5063535.73 & 20 \\
\hline prot_serine_sing_geom255 & -5063533.90 & -5063527.38 \\
\hline prot_serine_sing_geom258 & & 20 \\
\hline
\end{tabular}


Figure S3. Average distance between serine center of mass (COM) and ion.

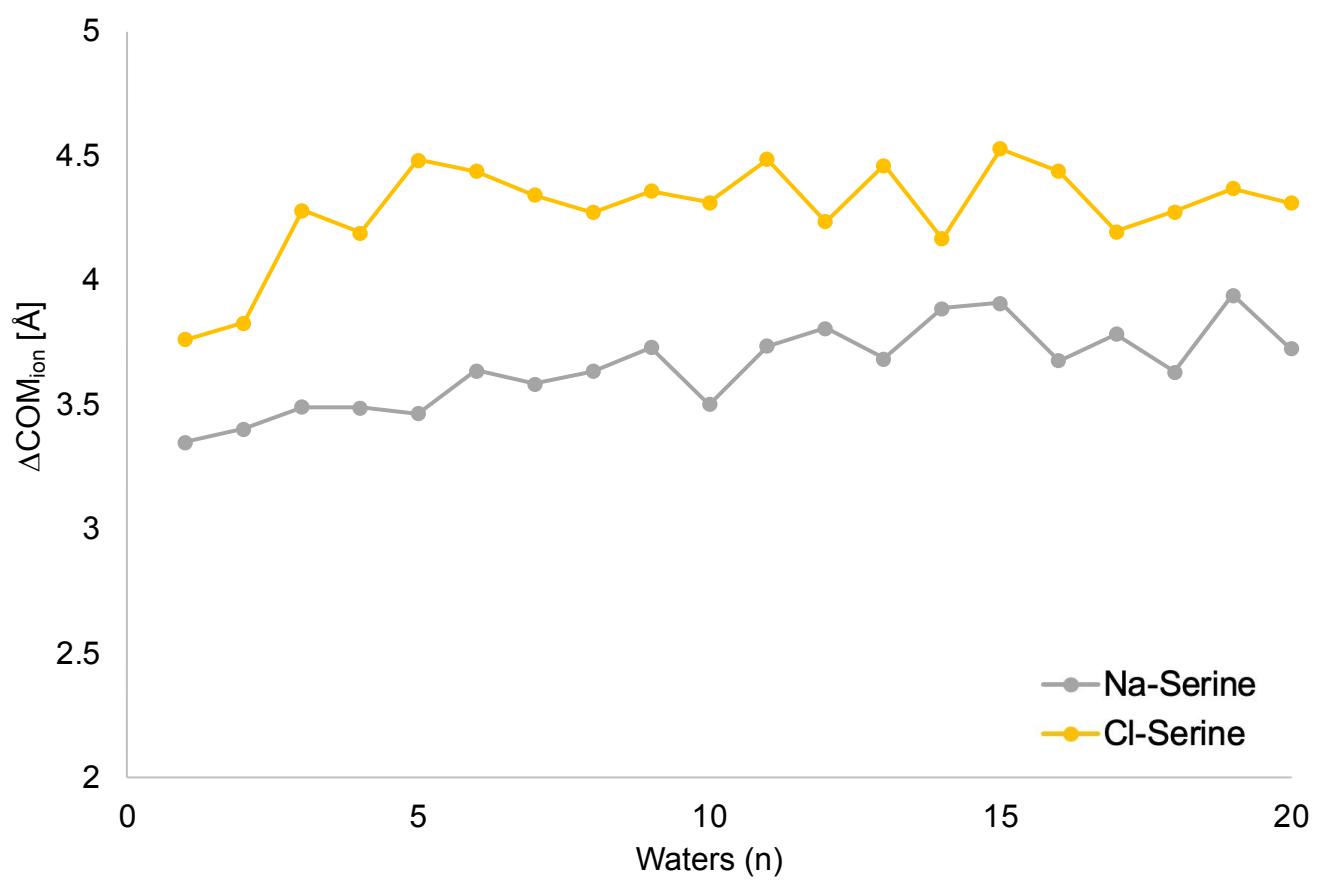


Table S7 - Energy and tautomeric state of the minimum energy structure of neutral serine, protonated serine, deprotonated from amine group serine and deprotonated from carboxylate group serine.

\begin{tabular}{|c|c|c|c|c|c|c|c|}
\hline $\begin{array}{l}\text { Number of } \\
\text { waters }\end{array}$ & Neutral & $\begin{array}{l}\text { Tautomeric } \\
\text { State* }\end{array}$ & $\begin{array}{l}\text { Protonated } \\
\text { Free Energy }\end{array}$ & $\begin{array}{l}\mathrm{H}^{+} \text {removed } \\
\text { from }-\mathrm{NH}_{3}\end{array}$ & $\begin{array}{l}\text { Tautomeric } \\
\text { State: }\end{array}$ & $\begin{array}{l}\mathrm{H}^{+} \text {removed } \\
\text { from }-\mathrm{COOH}\end{array}$ & $\begin{array}{l}\text { Tautomeric } \\
\text { State }\end{array}$ \\
\hline 0 & -1047702.9 & 0 & -1048608.8 & -1047702.9 & 0 & -1047702.9 & 0 \\
\hline 1 & -1248431.3 & 0 & -1249382.1 & -1248431.7 & 0 & -1248426.2 & 0 \\
\hline 2 & -1449183.2 & 0 & -1450146.9 & -1449159.3 & 0 & -1449169.4 & 0 \\
\hline 3 & -1649918.9 & 0 & -1650900.5 & -1649883.3 & 0 & -1649898.5 & 1 \\
\hline 4 & -1850661.6 & 0 & -1851645.9 & -1850597.2 & 0 & -1850642.1 & 2 \\
\hline 5 & -2051405.8 & 0 & -2052395.8 & -2051375.3 & 0 & -2051375.6 & 2 \\
\hline 6 & -2252150.8 & 2 & -2253147.1 & -2252091.0 & 0 & -2252136.6 & 2 \\
\hline 7 & -2452898.1 & 2 & -2453900.1 & -2452870.9 & 0 & -2452894.3 & 2 \\
\hline 8 & -2653636.4 & 2 & -2654630.6 & -2653582.0 & 0 & -2653612.0 & 2 \\
\hline 9 & -2854385.9 & 2 & -2855382.7 & -2854302.9 & 0 & -2854385.3 & 2 \\
\hline 10 & -3055128.2 & 2 & -3056140.7 & -3055067.2 & 0 & -3055126.1 & 2 \\
\hline 11 & -3255871.8 & 2 & -3256888.0 & -3255851.8 & 0 & -3255851.8 & 2 \\
\hline 12 & -3456619.8 & 2 & -3457614.5 & -3456593.1 & 0 & -3456573.6 & 3 \\
\hline 13 & -3657356.2 & 2 & -3658362.6 & -3657295.5 & 0 & -3657290.5 & 2 \\
\hline 14 & -3858118.4 & 2 & -3859108.3 & -3858039.1 & 0 & -3858073.0 & 2 \\
\hline 15 & -4058852.8 & 2 & -4059856.7 & -4058777.7 & 0 & -4058822.1 & 2 \\
\hline 16 & -4259590.7 & 2 & -4260591.4 & -4259511.6 & 0 & -4259563.7 & 2 \\
\hline 17 & -4460315.5 & 2 & -4461340.6 & -4460276.8 & 0 & -4460290.0 & 2 \\
\hline 18 & -4661072.4 & 2 & -4662096.8 & -4661038.1 & 0 & -4661028.1 & 2 \\
\hline 19 & -4861808.1 & 2 & -4862850.7 & -4861793.3 & 0 & -4861744.6 & 2 \\
\hline 20 & -5062579.1 & 2 & -5063589.2 & -5062504.1 & 0 & -5062543.8 & 2 \\
\hline
\end{tabular}

$¥ 0$ indicates the canonical form, 1 indicates the deprotonated form (proton from the carboxylate), and 2 indicates the zwitterionic form, 3 indicates all oxygen atoms have been deprotonated. 


\section{Molecular Coordinates}

Cartesian coordinates of all serine-water clusters can be found in the accompanying .xyz file. The names of each structure matches with the entries in the previous Tables S3-S6. They are in the following order: neutral serine, protonated serine, sodiated serine and finally chlorinated serine. These geometries can be opened with any chemistry GUI or viewer, including Mercury ((https://www.ccdc.cam.ac.uk/mercury/), which can be found online free of charge. 


\section{References}

1. Becke, A. D., Density-functional exchange-energy approximation with correct asymptotic behavior. Phys. Rev. A 1988, 38 (6), 3098-3100.

2. Perdew, J. P., Density-functional approximation for the correlation energy of the inhomogeneous electron gas. Phys. Rev. B 1986, 33 (12), 8822-8824.

3. Hunter, E. P. L.; Lias, S. G., Evaluated Gas Phase Basicities and Proton Affinities of Molecules: An Update. J. Phys. Chem. Ref. Data 1998, 27 (3), 413-656.

4. Grimme, S.; Antony, J.; Ehrlich, S.; Krieg, H., A consistent and accurate ab initio parametrization of density functional dispersion correction (DFT-D) for the 94 elements H-Pu. J. Chem. Phys. 2010, 132 (15), 154104.

5. Grimme, S.; Ehrlich, S.; Goerigk, L., Effect of the damping function in dispersion corrected density functional theory. J. Comp. Chem. 2011, 32 (7), 1456-1465.

6. Lee, C. T.; Yang, W. T.; Parr, R. G., Development of the Colle-Salvetti Correlation-Energy Formula into a Functional of the Electron Density. Phys. Rev. B 1988, 37 (2), 785-789.

7. Chai, J.-D.; Head-Gordon, M., Long-range corrected hybrid density functionals with damped atom-atom dispersion corrections. Phys. Chem. Chem. Phys. 2008, 10 (44), 6615-6620.

8. Zhao, Y.; Truhlar, D. G., The M06 suite of density functionals for main group thermochemistry, thermochemical kinetics, noncovalent interactions, excited states, and transition elements: two new functionals and systematic testing of four M06-class functionals and 12 other functionals. Theor. Chem. Acc. 2008, 120 (1-3), 215-241.

9. Armentrout, P. B.; Boles, G. C.; Ghiassee, M.; Berden, G.; Oomens, J., Infrared MultiplePhoton Dissociation Spectra of Sodiated Complexes of the Aliphatic Amino Acids. J. Phys. Chem. A 2021, 125 (29), 6348-6355.

10. Pham, H. D. M.; Boles, G. C.; Armentrout, P. B., Sodium Binding Interactions with Aliphatic Amino Acids: A Guided Ion Beam and Computational Study. J. Phys. Chem. A 2021, 125 (29), 63326347. 